\title{
Contemporary outcomes of continuous-flow left ventricular assist devices-a systematic review
}

\author{
Nicholas McNamara ${ }^{1,2,3}$, Harry Narroway ${ }^{4}$, Michael Williams ${ }^{1,2}$, John Brookes $^{1}$, James Farag ${ }^{1,3}$, \\ David Cistulli $^{1}$, Paul Bannon ${ }^{1,3}$, Silvana Marasco ${ }^{5,6}$, Evgenij Potapov ${ }^{7,8}$, Antonio Loforte ${ }^{9}$ \\ ${ }^{1}$ Cardiothoracic Surgical Department, Royal Prince Alfred Hospital, Sydney, Australia; ${ }^{2}$ Faculty of Health and Medicine, University of Sydney, \\ Sydney, Australia; ${ }^{3}$ Baird Institute of Applied Heart and Lung Research, Sydney, Australia; ${ }^{4}$ Department of Vascular Surgery, Gosford Hospital, \\ Gosford, Australia; ${ }^{5}$ Cardiothoracic Surgical Department, The Alfred, Melbourne, Australia; ${ }^{6}$ Department of Medicine and Surgery, Monash \\ University, Melbourne, Australia; ${ }^{7}$ Deutsches Zentrum für Herz Kreislauf Forschung (DZHK) - Standort Berlin/Charité, Berlin, Germany; \\ ${ }^{8}$ Department of Cardiothoracic and Vascular Surgery, Deutsches Herzzentrum Berlin (DHZB), Berlin, Germany; ${ }^{9}$ Division of Cardiac Surgery, \\ IRCCS Azienda Ospedaliero-Universitaria di Bologna, Bologna, Italy \\ Correspondence to: Nicholas McNamara. Cardiothoracic Surgical Department, Royal Prince Alfred Hospital, Sydney, Australia. \\ Email: nmcn8038@uni.sydney.edu.au.
}

Background: End stage heart failure is a major cause of morbidity and mortality, and its prevalence is expected to rise with the ageing population. For suitable patients, orthotopic heart transplantation remains the gold standard therapy, however, a paucity of donor organs has led to the development of left ventricular assist devices (LVAD). These devices can be utilized as either a bridge-to-transplant (BTT) or as an alternative to heart transplantation. While these devices can prolong life and improve quality of life, they are associated with a significant number of adverse events. We aim to systematically review the literature to quantify survival and the incidence of adverse events following implantation of continuous-flow LVADs (cf-LVAD).

Methods: A systematic review was performed to determine outcomes following implantation of a cf-LVAD. Primary outcomes were survival and frequency of adverse events (such as bleeding, infection, thrombosis, stroke and right ventricular failure). Secondary outcomes included quality of life and assessment of functional status.

Results: Sixty-three studies reported clinical outcomes of 9,280 patients. Survival after cf-LVAD varied between studies. Industry-funded trials generally reported better overall survival than the single- and multicenter case series, which showed significant variation. The largest registry report documented twelve, twentyfour and forty-eight-month survival rates of $82 \%, 72 \%$ and $57 \%$ respectively. The most commonly reported adverse events were gastrointestinal bleeding (GIB), device-related infection, neurological events and right heart failure (RHF). Bleeding, RHF and infection were the most frequent complications experienced by those supported with cf-LVAD, occurring in up to 35\%, $40 \%$ and $55 \%$ of patients, respectively. Quality of life as measured using the Kansas City Cardiomyopathy Questionnaire (KCCQ) and functional status as measured with the 6-minute walk test (6MWT) improved after cf-LVAD implantation with no decline evident two years after implantation.

Conclusions: The paucity of donor hearts has led to the development of left-ventricular assist devices as a BTT or as a destination therapy (DT). Outcomes after cf-LVAD implantation are excellent, with short-term survival comparable to heart transplantation, but long-term survival remains limited due to the incidence of post-implantation adverse events. Despite these complications, quality of life and functional status improve significantly post-implantation and remain improved over the long-term. This study demonstrates the potential benefits of cf-LVAD therapy whilst also identifying adverse events as an area of increased morbidity and mortality.

Keywords: Left ventricular assist device (LVAD); heart failure; systematic review; survival; adverse events 
Submitted Feb 01, 2021. Accepted for publication Mar 11, 2021.

doi: 10.21037/acs-2021-cfmcs-35

View this article at: http://dx.doi.org/10.21037/acs-2021-cfmcs-35

\section{Introduction}

The global prevalence of heart failure is estimated at 65 million cases and is expected to rise as the world population ages (1). End-stage heart failure (ESHF) is a major cause of morbidity and mortality and also contributes significantly to health care costs. Despite the introduction of highly effective medical therapies to reduce mortality and improve function, there remains a significant proportion of patients whose heart failure progresses despite optimal medical therapy. For suitable candidates with advanced and refractory heart failure, orthotopic heart transplant (OHT) remains the definitive therapy and can significantly extend survival and improve function and quality of life (2). However, due to a paucity of donor hearts, left ventricular assist devices (LVADs) have become a viable alternative. The first generation of these devices were volume displacement pumps that generated pulsatile flow (pfLVADs) analogous to endogenous cardiac contraction. Such devices were utilized as a bridge-to-transplant (BTT) but prolonged use was limited by poor mechanical durability and an unfavourable adverse event profile. The landmark REMATCH trial (3) established the superiority of the Heartmate XVE (Thoratec, Pleasanton, Calif.) over optimal medical therapy for patients with ESHF ineligible for OHT. This marked the beginning of LVAD use as destination therapy (DT). Second- and third-generation continuousflow (cf)-LVADs were designed to overcome the limitations of the first-generation devices. These devices utilize axial and centrifugal impellers, their smaller size permits implantation in adults and children with smaller body sizes, and their enhanced durability and reliability reduce device malfunction. Despite achieving improved survival and freedom from reoperation for device malfunction compared to pf-LVAD (4-6), cf-LVADs have their own challenges, primarily hemolysis, pump thrombosis, infection and gastrointestinal bleeding (GIB). The present systematic review aimed to examine the short and long-term clinical outcomes of cf-LVADs used as both BTT and DT.

\section{Methods}

\section{Search strategy and study selection}

Electronic searches were performed using Medline, Cochrane Central Register of Controlled Trials, Cochrane Database of Systematic Reviews, and the Database of Abstracts and Reviews of Effects for English-language studies on human subjects published between January 1, 2007 and December 5, 2020. The year 2007 was chosen as this is when contemporary data on CF-LVAD started to become available. Our methods adhered to the guidelines set forth in the Preferred Reporting Items for Systematic Reviews and Meta-Analyses: The PRISMA Statement (7). The search algorithm included a combination of Medical Subject Headings (MeSH) and keyword terms related to heart failure, left ventricular assist devices (LVADs) and outcomes of interest (such as survival and adverse effects) (Figure 1). Studies including both BTT and DT were included. The reference lists of selected studies were manually reviewed to identify any relevant studies that were potentially missed in the database search. Three reviewers (NM, MW, DC) independently screened the title and abstract of all records identified in the search. When the title and abstract provided insufficient detail to determine study relevance, a full-text copy of the article was reviewed. Before final inclusion, full-text copies of all selected articles were examined for eligibility.

\section{Inclusion and exclusion criteria}

Eligible studies were those reporting either survival or adverse event outcome data for patients who had undergone insertion of a cf-LVAD for heart failure. Where multiple studies reported on the same outcome for the same or overlapping patient cohorts, only the most comprehensive or most recent publication was included. The following exclusion criteria were applied: reviews or editorials, conference proceedings, studies with non-human participants, surgical techniques, pediatric studies, studies 


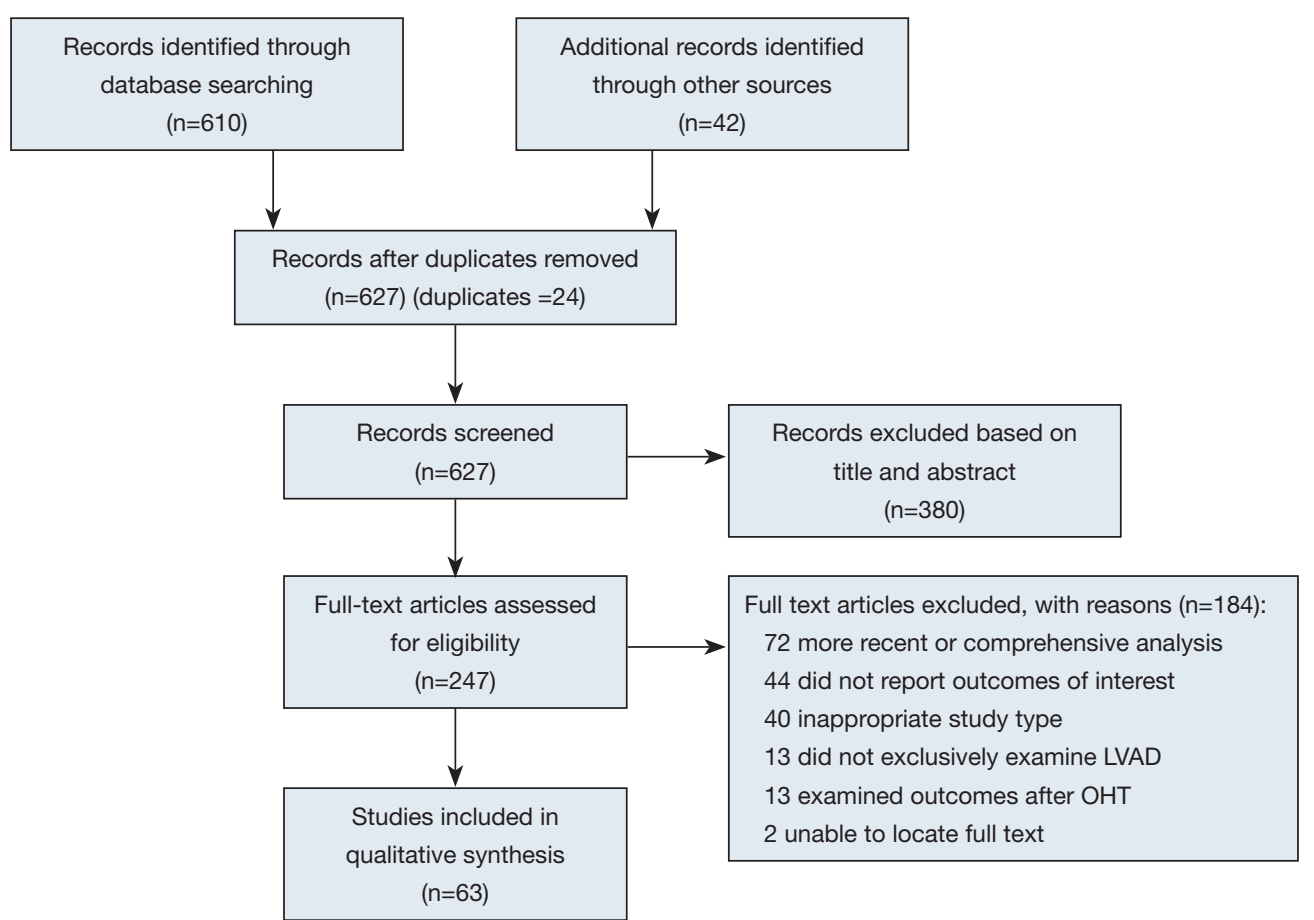

Figure 1 PRISMA diagram. LVAD, left ventricular assist device; OHT, orthotopic heart transplant.

that did not separate data for pulsatile and continuous flow devices, studies reporting on mechanical assist devices other than isolated implantable left ventricular assist devices, studies reporting on risk modeling, studies examining posttransplant outcomes in patients bridged with an LVAD, studies that were updated by newer publications and subanalyses of previously reported results.

\section{Study endpoints}

Primary outcomes were survival and frequency of adverse events (such as bleeding, infection, thrombosis, stroke and right ventricular failure). Secondary outcomes included quality of life and assessment of functional status.

\section{Data extraction and quality assessment}

Two independent reviewers (NM and MW) extracted data directly from publication texts, tables and figures. The following information was extracted from each study: number of patients implanted with cf-LVAD, device model, duration of follow-up or mean duration of support or defined time at risk (cumulative patient-years), patient demographics and outcome measures (survival, adverse events, OHT, functional status, quality of life measure). A third reviewer (JB) independently reviewed and confirmed all abstracted data. Disagreements were resolved by consensus. Due to the manner in which outcome data was extracted, each study was effectively treated as a case series regardless of the initial study design, and therefore an assessment of the quality of each study was not performed.

\section{Data synthesis}

Data were summarized in tabular form. Where data were reported separately for subgroups, a weighted estimate of the cumulative outcome value was calculated. When relevant data were only presented in graphic form, quantitative estimates were extracted and reported. Narrative synthesis of data was added to supplement the tables, describe data quality and provide a summary. Due to the high likelihood of patient data from single- and multicenter case series being duplicated in registry and trial publications, the authors chose to stratify studies by these 
three study types. Due to the significant heterogeneity between studies, pooling of data for meta-analysis was not deemed appropriate.

\section{Role of the funding source}

No funding sources were involved in the study design, data collection, data analysis, data interpretation or writing of the report. The corresponding authors had full access to all the data in the study and had final responsibility for the decision to submit for publication.

\section{Results}

A total of 627 publications were identified through electronic database searches and from other sources including reference lists (Figure 1). After exclusion of duplicate and irrelevant publications, a total of 63 relevant articles were included in the present review (Table 1). The majority of studies were observational, single- or multicenter case series. There were 14 industry-funded trials with 4,152 patients, and 46 single- and multi-center case series which included 5,128 patients; 41 of these were retrospective observational studies and five were prospective. Due to significant overlap between registry studies, industry-funded trials and case-series the authors decided to separate studies into these three categories for review.

Prospective registries tended to be used for numerous publications on individual outcomes or specific subgroup analyses, and therefore, there was significant overlap between the registry studies. For this reason, three registry reports were included for comparison: Goldstein et al. 2019 (68) was chosen for survival outcomes as it utilized the largest registry and includes most patients from the remaining registry studies. John et al. 2011 (67) was selected as a comparison for adverse event and functional outcomes as it was the largest study reporting these outcomes, although it was limited to the HeartMate II device utilized as BTT. Kormos et al. 2019 (69) was included for qualityof-life outcomes as these were not reported by the other two registry studies.

\section{Study characteristics}

Patient age was similar in the industry-funded studies (weighted mean patient age 57 years, range 49 to 66 years), single- and multi-center case series (53 years, range 35 to 69 years) and the largest registry report $(56 \pm 12$ years).
Comparing studies based on indication for LVAD implantation, patients were youngest in the studies examining BTT and oldest in those examining DT, an expected finding as many DT patients are not considered for OHT based on their age (Table 2). The majority of LVAD recipients were male: $80 \%$ in industry-funded trials, $79 \%$ in case series and $79 \%$ in the registry report, and this did not change with indication for LVAD implantation.

There was significant heterogeneity between studies in regard to the number of participants, device implanted, indication for support, proportion of patients with ischemic cardiomyopathy (ICM), Interagency Registry for Mechanically Assisted Circulatory Support (INTERMACS) profile and duration of follow up (Table 1). Cohorts ranged from eight to 1,028 participants, with 30 studies reporting outcomes for less than 100 participants. The proportion of participants with ICM ranged from $6 \%$ to $80 \%$. The HeartMate II (Thoratec, Pleasanton, Calif.) and HeartWare HVAD (HeartWare Inc., Framingham, MA) were the most frequently investigated devices. The majority of the industry-funded studies examined a single device, whereas the single- and multi-center case series tended to pool outcomes for all devices implanted at their institution(s). Follow up was largely reported as mean and standard deviation with a few studies reporting median and interquartile range or mean/median duration of support or cumulative time on support (patient-years). A summary of the proportion of patients in each study who experienced an adverse event is presented in Table 3. The significant heterogeneity of duration of follow up and reporting made a comparison of the incidence of adverse events difficult, therefore we chose to also present the adverse event rate expressed as events per patient-year (Table 4).

\section{Outcome analysis}

\section{Survival}

Thirteen industry-funded trials and 34 single- and multicenter case series reported survival following cf-LVAD implantation (Table 5). Although survival is an objective measure, significant differences in the duration of follow up, censoring of patients at time of transplant and patient loss to follow up made this outcome difficult to report. Actuarial survival was the single measure of survival with the most uniform definition across the studies and was chosen as the outcome measure for comparison. Most studies reported estimated actuarial survival in the range of one to twelve months, with few studies reporting survival 


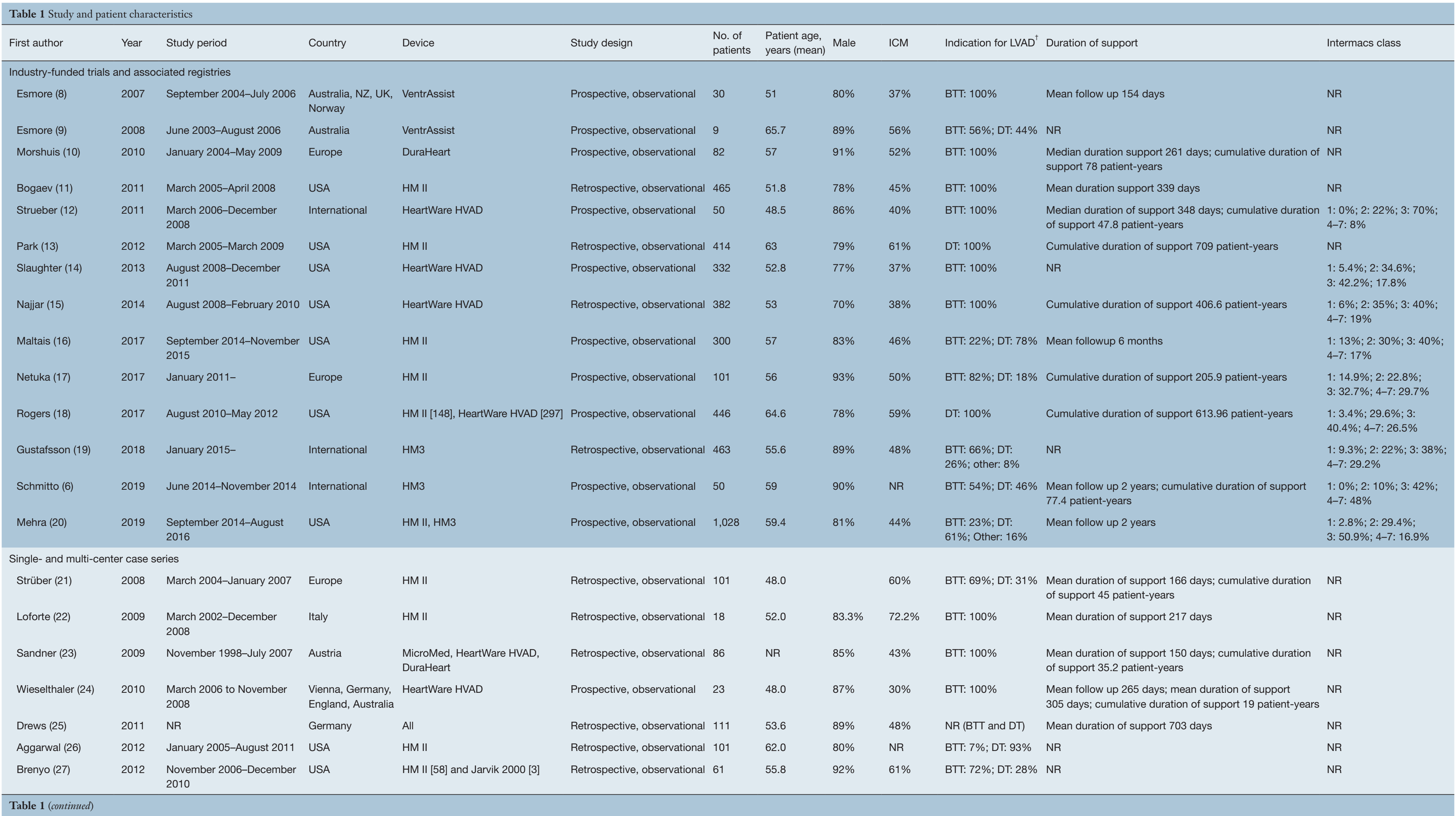




\begin{tabular}{|c|c|c|c|c|c|c|c|c|c|c|c|c|}
\hline First author & Year & Study period & Country & Device & Study design & $\begin{array}{l}\text { No. of } \\
\text { patients }\end{array}$ & $\begin{array}{l}\text { Patient age, } \\
\text { years (mean) }\end{array}$ & Male & ICM & Indication for LVAD ${ }^{\dagger}$ & Duration of support & Intermacs class \\
\hline Menon (28) & 2012 & August 2008-February 2011 & Germany & HM II & Retrospective, observational & 40 & 58.1 & $65 \%$ & $80 \%$ & $\begin{array}{l}\text { BTT: 63\%; DT: } \\
\text { 23\%; other: } 15 \%\end{array}$ & $\begin{array}{l}\text { Mean follow up } 245 \text { days; cumulative duration of support } \\
29 \text { patient-years }\end{array}$ & NR \\
\hline Ozbaran (29) & 2012 & $\begin{array}{l}\text { December 2010-August } \\
2011\end{array}$ & Turkey & HeartWare HVAD & Retrospective, observational & 10 & 51.8 & $90 \%$ & $30 \%$ & BTT: 80\%; DT 20\% & Mean duration of support 251 days & $\begin{array}{l}\text { 1: } 10 \% ; 2: 70 \% ; 3: 20 \% ; \\
\text { 4-7:0\% }\end{array}$ \\
\hline Raasch (30) & 2012 & Jan 2006-Feb 2011 & USA & HM II [47] or Jarvik 2000 [14] & Retrospective, observational & 61 & 56.0 & $67 \%$ & $39 \%$ & BTT: 44\%; DT: 56\% & NR & NR \\
\hline Sorensen (31) & 2012 & $\begin{array}{l}\text { September 2002- } \\
\text { September } 2010\end{array}$ & USA & HM II [30], Jarvik 2000 [35] & Retrospective, observational & 65 & 54.0 & $75 \%$ & $49 \%$ & ВТТ: $100 \%$ & NR & NR \\
\hline Yuan (32) & 2012 & June 2000-March 2012 & USA & HM II & Retrospective, observational & 87 & 50.0 & $74 \%$ & $27 \%$ & $\begin{array}{l}\text { BTT: } 51 \% \text {; DT: } \\
\text { 30\%; Other } 19 \%\end{array}$ & Mean duration of support 303 days & NR \\
\hline Dell'Aquila (33) & 2013 & July 2009-November 2011 & Germany & HeartWare HVAD & Retrospective, observational & 50 & 50.6 & $78 \%$ & $24 \%$ & ВТТ: $100 \%$ & $\begin{array}{l}\text { Mean duration of support } 285 \text { days; cumulative duration } \\
\text { of support } 30 \text { patient-years }\end{array}$ & $\begin{array}{l}\text { 1: } 22 \% ; 2: 10 \% ; 3: 20 \% ; \\
4-7: 48 \%\end{array}$ \\
\hline Forest (34) & 2013 & June 2006-December 2011 & USA & $\begin{array}{l}\text { HM II [58], HeartWare HVAD [9] } \\
\text { and VentrAssist [4] }\end{array}$ & Retrospective, observational & 71 & 56.0 & $77 \%$ & $31 \%$ & BTT: 62\%; DT: 38\% & $\begin{array}{l}\text { Mean duration of support } 359 \text { days; cumulative duration } \\
\text { of support } 68 \text { patient-years }\end{array}$ & NR \\
\hline Kutty (35) & 2013 & May 2005-December 2010 & UK & $\begin{array}{l}\text { HeartWare HVAD [8], } \\
\text { VentrAssist [21] }\end{array}$ & Retrospective, observational & 29 & 45.0 & $86 \%$ & $31 \%$ & $\begin{array}{l}\text { BTT: } 41 \% \text {; } \\
\text { other: } 59 \%\end{array}$ & Mean duration of support 327 days & $\begin{array}{l}\text { 1: } 0 \% ; 2: 28 \% ; 3: 59 \% ; \\
4-7: 14 \%\end{array}$ \\
\hline Lok (36) & 2013 & $\begin{array}{l}\text { March 2006-December } \\
2011\end{array}$ & Netherlands & HM II & Prospective, observational & 85 & 45.0 & $73 \%$ & $28 \%$ & ВТТ: $100 \%$ & $\begin{array}{l}\text { Median suration of support } 387 \text { days; cumulative duration } \\
\text { of support } 109 \text { patient-years }\end{array}$ & $\begin{array}{l}\text { 1:25\%; } 2: 75 \% ; 3: 0 \% \\
\text { 4-7: } 0 \%\end{array}$ \\
\hline Meyer (37) & 2013 & February 2004-2009 & Germany & $\begin{array}{l}\text { HM II [74] and HeartWare HVAD } \\
\text { [41] }\end{array}$ & Retrospective, observational & 115 & 50.0 & $88 \%$ & $39 \%$ & BTT: 92\%; DT: 8\% & NR & NR \\
\hline Saeed (39) & 2013 & $\begin{array}{l}\text { February 2005-February } \\
2006\end{array}$ & Europe & CorAide LVAS & Prospective, observational & 21 & 63.0 & $85 \%$ & $42 \%$ & NR (BTT and DT) & $\begin{array}{l}\text { Median duration of support } 192 \text { days; cumulative duration } \\
\text { of support } 17 \text { patient-years }\end{array}$ & $\begin{array}{l}1: 0 \% ; 2: 28 \% ; 3: 72 \% \\
4-7: 0 \%\end{array}$ \\
\hline Sakaguchi (40) & 2013 & $\begin{array}{l}\text { October 2008-October } \\
2011\end{array}$ & Japan & DuraHeart & Retrospective, observational & 23 & 35.1 & $74 \%$ & $13 \%$ & ВТТ: $100 \%$ & $\begin{array}{l}\text { Median duration of support } 559 \text { days; cumulative duration } \\
\text { of support } 35 \text { patient-years }\end{array}$ & $\begin{array}{l}\text { 1: } 17 \% ; 2: 48 \% ; 3: 35 \% \\
4-7: 0 \%\end{array}$ \\
\hline Özalp (41) & 2014 & $\begin{array}{l}\text { January 2009-September } \\
2013\end{array}$ & UK & $\begin{array}{l}\text { VentrAssist [6] and HeartWare } \\
\text { HVAD [96] }\end{array}$ & Retrospective, observational & 102 & 47.0 & $87 \%$ & $39 \%$ & ВTT: $100 \%$ & $\begin{array}{l}\text { Median follow up } 628 \text { days; median duration of support } \\
462 \text { days }\end{array}$ & $\begin{array}{l}\text { 1: 6\%; 2: } 36 \% ; 3: 25 \% ; \\
4-7: 33 \%\end{array}$ \\
\hline Sabashnikov (42) & 2014 & July 2007-August 2013 & UK & $\begin{array}{l}\text { HM II [72] and HeartWare } \\
\text { HVAD [67] }\end{array}$ & Retrospective, observational & 139 & 44.0 & $83 \%$ & $11 \%$ & ВТТ: $100 \%$ & Mean duration of support 514 days & $\begin{array}{l}\text { 1: } 20 \% ; 2: 40 \% ; 3: 30 \% ; \\
\text { 4-7: } 10 \%\end{array}$ \\
\hline Takeda (43) & 2014 & March 2004-June 2010 & USA & $\begin{array}{l}\text { HM II [117], VentrAssist [9], } \\
\text { DuraHeart [6], DeBakey [6] }\end{array}$ & Retrospective, observational & 140 & 54.7 & $79 \%$ & $36 \%$ & BTT: 82\%; DT: 18\% & Mean duration of support 438 days & NR \\
\hline Yoshioka (44) & 2014 & 2005 to 2010 & Japan & Jarvik 2000 & Retrospective, observational & 8 & 55.0 & $75 \%$ & $13 \%$ & BTT: 75\%; DT: 25\% & NR & NR \\
\hline Ertugay (45) & 2015 & August 2012-August 2014 & Turkey & HM II & Retrospective, observational & 28 & 51.2 & $97 \%$ & $36 \%$ & BTT: 86\%; DT: 14\% & Mean duration of support 326 days & $\begin{array}{l}\text { 1: } 0 \% ; 2: 21 \% ; 3: 50 \% \\
4-7: 29 \%\end{array}$ \\
\hline Hata (46) & 2015 & $\begin{array}{l}\text { January 2009-September } \\
2013\end{array}$ & Japan & $\begin{array}{l}\text { EVAHEART [14], HM II [14], } \\
\text { DuraHeart [4] }\end{array}$ & Retrospective, observational & 32 & 40.2 & $88 \%$ & $13 \%$ & ВТТ: $100 \%$ & $\begin{array}{l}\text { Median duration of support } 563 \text { days; cumulative duration } \\
\text { of support } 33.9 \text { patient-years }\end{array}$ & $\begin{array}{l}\text { 1: } 0 \% ; 2: 38 \% ; 3: 62 \% ; \\
\text { 4-7:0\% }\end{array}$ \\
\hline lacovoni (47) & 2015 & January 2006-May 2012 & Italy & INCOR & Retrospective, observational & 42 & 56.0 & $93 \%$ & $45 \%$ & BTT: 86\%; DT: $14 \%$ & $\begin{array}{l}\text { Mean duration of support } 525 \text { days; cumulative duration } \\
\text { of support } 60 \text { patient-years }\end{array}$ & $\begin{array}{l}\text { 1: } 41 \% ; 2: 33 \% ; 3: 19 \% ; \\
\text { 4-7: } 7 \%\end{array}$ \\
\hline Kimura (48) & 2015 & April 2011-August 2013 & Japan & DuraHeart [10], EVAHEART [21] & Retrospective, observational & 31 & 39.7 & $84.0 \%$ & $6.0 \%$ & ВТТ: $100 \%$ & $\begin{array}{l}\text { Mean follow up } 483 \text { days; cumulative duration of support } \\
41 \text { patient-years }\end{array}$ & $\begin{array}{l}\text { 1: } 19 \% ; 2: 45 \% ; 3: 32 \% ; \\
4-7: 3 \%\end{array}$ \\
\hline
\end{tabular}




\begin{tabular}{|c|c|c|c|c|c|c|c|c|c|c|c|c|}
\hline First author & Year & Study period & Country & Device & Study design & $\begin{array}{l}\text { No. of } \\
\text { patients }\end{array}$ & $\begin{array}{l}\text { Patient age, } \\
\text { years (mean) }\end{array}$ & Male & ICM & Indication for LVAD & Duration of support & Intermacs class \\
\hline Lushaj (49) & 2015 & January 2008-June 2014 & USA & HM II, HeartWare HVAD & Retrospective, observational & 128 & 57.2 & $84 \%$ & $23 \%$ & BTT: 66\%; DT: $34 \%$ & 24 months (censored at transplant or death) & $\begin{array}{l}\text { 1: } 28 \% ; 2: 28 \% ; 3: 14 \% \text {; } \\
\text { 4-7:30\% }\end{array}$ \\
\hline Ammirati (50) & 2016 & $\begin{array}{l}\text { January 2006-February } \\
2012\end{array}$ & Italy & $\begin{array}{l}\text { MicroMed DeBakey, Berlin Heart } \\
\text { Incor, HM II, HeartWare HVAD }\end{array}$ & Prospective, observational & 49 & 54.0 & $90 \%$ & $39 \%$ & $\begin{array}{l}\text { BTT: 45\%; DT: } \\
\text { 25\%; other: } 30 \%\end{array}$ & NR & NR \\
\hline Daneshmand (51) & 2015 & $\begin{array}{l}\text { January 2005-December } \\
2012\end{array}$ & USA & HM II & Retrospective, observational & 146 & 67.0 & $74 \%$ & $62 \%$ & DT: $100 \%$ & NR & $\begin{array}{l}\text { 1: } 9 \% ; 2: 46 \% ; 3: 27 \% \\
\text { 4-7: } 18 \%\end{array}$ \\
\hline John (52) & 2016 & June 1, 2005-June 30, 2014 & USA & HM II & Retrospective, observational & 278 & 57.2 & $81 \%$ & $58 \%$ & ВТТ: 79\%; DT: 21\% & $\begin{array}{l}\text { Median suration of support } 469 \text { days; cumulative duration } \\
\text { of support } 479 \text { patient-years }\end{array}$ & NR \\
\hline Sileshi (54) & 2016 & $\begin{array}{l}\text { January 2013-December } \\
2014\end{array}$ & USA & HeartWare HVAD & Retrospective, observational & 81 & 52.0 & $77 \%$ & $43 \%$ & ВТТ: $100 \%$ & Mean follow up 180 days & NR \\
\hline Xuereb (55) & 2016 & March 2006 to May 2015 & USA & HMII, HeartWare HVAD & Retrospective, observational & 240 & 54.5 & $75 \%$ & $37.90 \%$ & BTT: 53\%; DT: $47 \%$ & NR & NR \\
\hline Centofanti (56) & 2017 & $\begin{array}{l}\text { November 2010-March } \\
2016\end{array}$ & Italy & $\begin{array}{l}\text { HeartWare HVAD [31], Jarvik } 2000 \\
\text { [21], HM3 [1] }\end{array}$ & Retrospective, observational & 32 & 69.0 & $84 \%$ & $53 \%$ & $\begin{array}{l}\text { BTT: } 34 \% \text {; DT: } \\
\text { 41\%; other: } 25 \%\end{array}$ & Mean follow up 576 days & $\begin{array}{l}\text { 1: } 0 \% ; 2: 16 \% ; 3: 72 \% \\
\text { 4-7: } 12 \%\end{array}$ \\
\hline Hanke (57) & 2017 & May 2009-December 2015 & Germany & HeartWare HVAD & Retrospective, observational & 102 & NR & $78 \%$ & $26 \%$ & $\mathrm{NR}$ (BTT and DT) & NR & NR \\
\hline Steffen (58) & 2017 & October 2004-June 2013 & USA & HM II & Retrospective, observational & 285 & 55.0 & $81 \%$ & NR & ВТТ: $100 \%$ & $\begin{array}{l}\text { Mean follow up } 533 \text { days; mean duration of support } \\
216 \text { days; cumulative duration of support } 315 \text { patient-years }\end{array}$ & NR \\
\hline Tozzi (59) & 2017 & November 2015-June 2016 & Switzerland & HM3 & Prospective, observational & 10 & 57.3 & $90 \%$ & $70 \%$ & ВTT: 70\%; DT 30\% & NR & $\begin{array}{l}\text { 1: } 20 \% ; 2: 30 \% ; 3: 30 \% \text {; } \\
\text { 4-7:20\% }\end{array}$ \\
\hline Carrozzini (60) & 2018 & $\begin{array}{l}\text { January 2012-December } \\
2016\end{array}$ & Italy & $\begin{array}{l}\text { Heartware HVAD [31], Jarvik } 2000 \\
\text { [21], HM3 [1] }\end{array}$ & Retrospective, observational & 53 & 52.0 & $89.0 \%$ & $45.0 \%$ & ВTТ: $100 \%$ & Median duration of support 150 days & $\begin{array}{l}\text { 1: } 42 \% ; 2: 30 \% ; 3: 8 \% \\
4-7: 21 \%\end{array}$ \\
\hline Tahsili-Fahadan (61) & 2018 & May 2005-December 2013 & USA & HM II [326], HeartWare HVAD [46] & Retrospective, observational & 372 & NR & $80 \%$ & $58 \%$ & ВТТ: 68\%; DT: 32\% & $\begin{array}{l}\text { Mean follow up } 664 \text { days; mean duration of support } \\
623 \text { days }\end{array}$ & NR \\
\hline Volkovicher (62) & 2018 & $\begin{array}{l}\text { November 2003-March } \\
2016\end{array}$ & USA & HM II [403], HeartWare HVAD [123] & Retrospective, observational & 526 & 54.7 & $78 \%$ & $45 \%$ & NR (BTT and DT) & Cumulative duration of support 871 patient-years & NR \\
\hline Yin (63) & 2018 & 2004 to 2017 & USA & Unspecified axial and centrifugal & Retrospective, observational & 351 & 59.0 & $82 \%$ & $45 \%$ & $\begin{array}{l}\text { BTT: } 51 \% \text {; DT: } \\
\text { 34\%; other: } 15 \%\end{array}$ & Median follow up 196 days & $\begin{array}{l}\text { 1: } 12 \% ; 2: 15 \% ; 3: 45 \% ; \\
\text { 4-7: } 28 \%\end{array}$ \\
\hline Braun (64) & 2019 & 1993-2013 & $\begin{array}{l}\text { Europe (Denmark, } \\
\text { Finland, Norway, } \\
\text { Sweden) }\end{array}$ & HM II and HeartWare HVAD & Retrospective, observational & 244 & 54.1 & $83 \%$ & $39 \%$ & ВТT: $87 \%$; DT 13\% & Mean follow up 195 days & $\begin{array}{l}\text { 1: } 12 \% ; 2: 28 \% ; 3: 43 \% ; \\
\text { 4-7: } 17 \%\end{array}$ \\
\hline Jorde (65) & 2019 & $\begin{array}{l}\text { January 2012-December } \\
2016\end{array}$ & USA & HM II & Retrospective, observational & 124 & 54.4 & $75 \%$ & $36 \%$ & ВТТ: 28\%; DT: $72 \%$ & $\begin{array}{l}\text { Mean follow up } 365 \text { days; cumulative duration of follow } \\
\text { up } 85.5 \text { patient-years }\end{array}$ & $\begin{array}{l}\text { 1: } 2 \% ; 2: 11 \% ; 3: 84 \% \\
4-7: 3 \%\end{array}$ \\
\hline Kyvernitakis (66) & 2019 & January 2006-July 2016 & USA & $\begin{array}{l}\text { HM II [170] and HeartWare } \\
\text { HVAD [42] }\end{array}$ & Retrospective, observational & 212 & 60 (median) & $80 \%$ & $58 \%$ & BTT: 59\%; DT: $41 \%$ & Median duration of support 257 days & $\begin{array}{l}\text { 1: } 25 \% ; 2: 49 \% ; 3: 12 \% \text {; } \\
\text { 4-7: } 14 \%\end{array}$ \\
\hline \multicolumn{13}{|l|}{ Registry reports } \\
\hline John (67) & 2011 & 2005-2011 & $\begin{array}{l}\text { INTERMACS } \\
\text { registry }\end{array}$ & HM II & Registry report & 1,982 & NR & $77 \%$ & NR & ВТТ: $100 \%$ & Mean duration of support 378 days & $\begin{array}{l}\text { 1: } 17 \% ; 2: 44 \% ; 3: 20 \% ; \\
\text { 4-7: } 19 \%\end{array}$ \\
\hline Goldstein (68) & 2019 & Inception-December 2017 & International & All approved devices & Registry report & 16,286 & 56 & $79 \%$ & $44 \%$ & $\begin{array}{l}\text { BTT: } 29 \% \text {; DT: } \\
\text { 42\%; other: } 27 \%\end{array}$ & NR & NR \\
\hline Kormos (69) & 2019 & April 2008-December 2017 & USA & All FDA-approved devices & Registry report & 18,539 & 57.1 & $79 \%$ & $81 \%$ & ВТT: 57\%; DT: 43\% & Mean duration of support 600 days & $\begin{array}{l}\text { 1: } 14 \% ; 2: 37 \% ; 3: 33 \% ; \\
\text { 4-7: } 16 \%\end{array}$ \\
\hline
\end{tabular}

${ }^{\dagger}$ indication for LVAD Other: bridge-to-recovery, bridge-to-decision. LVAD, left ventricular assist device; BTT, bridge-to-transplant; DT, destination therapy; ICM, ischemic cardiomyopathy. 


\begin{tabular}{|c|c|c|c|}
\hline \multirow{2}{*}{$\begin{array}{l}\text { Indication } \\
\text { for LVAD }\end{array}$} & \multicolumn{3}{|c|}{ Patient age (years) } \\
\hline & Industry & Case series* & All \\
\hline BTT & 53 & 49 & 51 \\
\hline Combined & 64 & 67 & 64 \\
\hline DT & 58 & 55 & 56 \\
\hline All patients & 57 & 54 & 56 \\
\hline
\end{tabular}

outcomes beyond two years. The International Registry for Mechanically Assisted Circulatory Support (IMACS) Third Annual Report published 1-, 6-, 12-, 24-, 36-, and 48 -month survival rates of $95 \%, 87 \%, 82 \%, 72 \%, 62 \%$ and $57 \%$, respectively (68). These values represent the most recent and comprehensive analysis of survival in patients supported with cf-LVADs. Industry-funded trials reported survival outcomes on par with those of the IMACS reports, while single- and multi-center case series showed the largest variation in values, with some of the poorest survival outcomes often coming from small trials with a large proportion of patients with DT indication.

\section{Bleeding}

Bleeding is a frequently reported adverse event and is often described as early or late to differentiate surgical from nonsurgical bleeding. Nine industry-funded trials and 26 singleand multi-center case series reported on the incidence of bleeding post cf-LVAD implantation (Table 3). Within the literature, the proportion of patients experiencing bleeding that required surgery, varied from $0 \%$ to $45 \%$ in case series and industry trials, compared with only $7 \%$ in the 1,496 patients who received the HeartMate II as BTT reported by John et al. 2011 (67). Reasons for such variance include differences in surgical technique (such as the use of lateral thoracotomy for implantation), postoperative anticoagulant regimens and patient risk factors (such as renal function). Examining the studies that reported bleeding event rates (Table 4), the industry-funded trials reported a fairly consistent rate of 0.13 to 0.26 events per patient-year, whereas the single- and multi-center case series reported rates that were generally twice that (range 0.16 to 0.59 events per patient-year).

Continuous flow LVAD support has also been associated with the development of a bleeding diathesis that manifests as late bleeding, predominantly GIB $(70,71)$. Industryfunded studies reported an incidence of GIB ranging from $6 \%$ to $35 \%$ which is similar to the $0 \%$ to $34 \%$ reported in case series. Examining studies that reported this outcome as an event rate, this outcome occurs with a frequency of between 0.0 and 0.71 events per patient-year. Greater variation was seen in the case-series compared with the industry trials.

\section{Neurological events}

Neurological events including ischemic stroke, hemorrhagic stroke, and transient ischemic attack (TIA) are relatively common after cf-LVAD implantation and can be a cause of significant morbidity and mortality. Eleven industryfunded trials and 22 single- and multi-center case series reported the incidence of neurological events after LVAD implantation (Table 3). The risk of ischemic and hemorrhagic stroke ranged from $0 \%$ to $26 \%$, and $0 \%$ to $16 \%$, respectively. Follow up in these studies ranged from approximately six months to two years, with a higher risk of stroke generally associated with prolonged duration of device support. This observation is in line with the findings of the fifth INTERMACS annual report, which demonstrated the 6-, 12-, and 24-month risk of stroke to be $7 \%, 11 \%$ and $17 \%$, respectively (72).

\section{Infection}

Despite the smaller diameter power cable compared to the first-generation pf-LVAD devices, the cf-LVAD driveline remains a source of entry for bacteria with the potential for developing driveline infection (DLI), pump pocket infection or sepsis. Ten industry-funded trials and twenty single- and multi-center case series (Table 3) reported outcomes for device-related infection and sepsis. DLI was one of the most common complications after LVAD implantation, occurring in $5 \%$ to $44 \%$ of patients. Despite the high rates of DLI, pump pocket infection rates were much lower, ranging from $0 \%$ to $22 \%$ among the various studies. Reported incidence of sepsis was almost as varied as that of DLI, with rates of sepsis ranging from $0 \%$ to $33 \%$. When reported as events per patient-year, DLI ( 0.13 to 1.27$)$ was more common than sepsis $(0.07$ to 0.46$)$ and pump pocket infection (0.01 to $0.07)$.

\section{Pump thrombosis}

Six industry-funded trials and eight single- and multi- 


\begin{tabular}{|c|c|c|c|c|c|c|c|c|c|c|c|c|c|c|}
\hline \multirow[b]{2}{*}{ First author } & \multirow[b]{2}{*}{ Year } & \multirow{2}{*}{$\begin{array}{l}\text { No. of } \\
\text { patients }\end{array}$} & \multirow[b]{2}{*}{ Indication for LVAD } & \multirow{2}{*}{$\begin{array}{l}\text { Support duration } \\
\text { (patient-years) }\end{array}$} & \multicolumn{2}{|l|}{ Bleeding } & \multicolumn{2}{|c|}{ Neurological } & \multirow{2}{*}{$\begin{array}{l}\text { Device malfunction } \\
\text { Thrombosis requiring } \\
\text { exchange }\end{array}$} & \multicolumn{3}{|l|}{ Infection } & \multicolumn{2}{|c|}{ Right heart failure } \\
\hline & & & & & $\begin{array}{l}\text { Requiring } \\
\text { surgery }\end{array}$ & GIB & $\begin{array}{l}\text { Ischaemic } \\
\text { stroke }\end{array}$ & $\begin{array}{l}\text { Haemorrhagic } \\
\text { stroke }\end{array}$ & & DLI & Sepsis & Pocket infection & RVAD & Inotrope \\
\hline \multicolumn{15}{|c|}{ Industry-funded trials and associated registries } \\
\hline Bogaev (11) & 2011 & 465 & ВТТ: $100 \%$ & 431.2 & $21.50 \%$ & & $4.90 \%$ & $5.10 \%$ & & & $21 \%$ & & $6.2 \%$ & $19.7 \%$ \\
\hline Strueber (12) & 2011 & 50 & ВТТ: $100 \%$ & 47.8 & $20 \%$ & & $4 \%$ & $8 \%$ & $2 \%$ & $18 \%$ & $10 \%$ & & $6 \%$ & $6.0 \%$ \\
\hline Park (13) & 2012 & 414 & DT: $100 \%$ & 709 & $22.90 \%$ & & $8.00 \%$ & $6.80 \%$ & $2.40 \%$ & $28.30 \%$ & $30.40 \%$ & $7.70 \%$ & $5.30 \%$ & $21.50 \%$ \\
\hline Najjar (15) & 2014 & 382 & ВТТ: 100\% & 406.6 & $14.90 \%$ & $15.40 \%$ & $5.20 \%$ & $8.30 \%$ & $4.20 \%$ & $19.60 \%$ & $18.80 \%$ & & $3.90 \%$ & $29.8 \%$ \\
\hline Maltais (16) & 2017 & 300 & BTT: 22\%; DT: 78\% & Mean 180 days follow up & $16 \%$ & $21 \%$ & $4 \%$ & $2.70 \%$ & $4.30 \%$ & $5 \%$ & & & $5 \%$ & $11.6 \%$ \\
\hline Netuka (17) & 2017 & 101 & BTT: 82\%; DT 18\% & 205.96 & & & $5 \%$ & $4 \%$ & & & & & & \\
\hline Rogers (18) & 2017 & 446 & DT: $100 \%$ & 613.96 & $13.60 \%$ & $34.70 \%$ & $14.40 \%$ & $11.30 \%$ & $7.90 \%$ & $18.20 \%$ & $20.90 \%$ & - & $2.90 \%$ & $31.70 \%$ \\
\hline Gustafsson (19) & 2018 & 463 & BTT: 66\%; DT: 26\%; other: 8\% & NR & $10 \%$ & $6 \%$ & $3.90 \%$ & $1.50 \%$ & & $11.70 \%$ & $9.10 \%$ & & $6.70 \%$ & $8.0 \%$ \\
\hline Schmitto (6) & 2019 & 50 & BTT: 54\%; DT: 46\% & 77.4 & $16 \%$ & $20 \%$ & $16 \%$ & $8 \%$ & - & $24 \%$ & $22 \%$ & & $4 \%$ & $14.0 \%$ \\
\hline Mehra (20) & 2019 & 1,020 & BTT: 23\%; DT: 61\%; other: 16\% & Mean follow up 2 years & $13.60 \%$ & $27.60 \%$ & $9.20 \%$ & $6.70 \%$ & $5.90 \%$ & $21.40 \%$ & $15 \%$ & & $4.10 \%$ & $27.1 \%$ \\
\hline \multicolumn{15}{|c|}{ Single- and multi-center case series } \\
\hline Strüber (21) & 2008 & 101 & ВTT: 69\%; DT: 31\% & 44.6 & - & - & - & - & - & $20.80 \%$ & - & $3 \%$ & - & - \\
\hline Loforte (22) & 2009 & 18 & ВТТ: $100 \%$ & Mean 217 days support & $33.30 \%$ & - & - & $5.60 \%$ & - & $11.10 \%$ & - & - & $11.10 \%$ & - \\
\hline Wieselthaler (24) & 2010 & 23 & ВТТ: $100 \%$ & 19 & $21.7 \%$ & $13 \%$ & $4.30 \%$ & $4.30 \%$ & $6.70 \%$ & $34.80 \%$ & $13 \%$ & - & $4.30 \%$ & - \\
\hline Aggarwal (26) & 2012 & 101 & ВTT: 7\%; DT: 93\% & NR & - & 22.8 & - & - & - & - & - & - & - & - \\
\hline Brenyo (27) & 2012 & 61 & BTT: 72\%; DT: 28\% & NR & - & - & $11 \%$ & - & - & - & - & - & - & - \\
\hline Menon (28) & 2012 & 40 & BTT: 63\%; DT: 23\%; other: 15\% & 29 & $15 \%$ & $7.50 \%$ & $5 \%$ & - & - & - & - & - & - & - \\
\hline Ozbaran (29) & 2012 & 10 & BTT: 80\%; DT 20\% & Mean 251 days support & $30 \%$ & - & - & $1 \%$ & - & - & - & - & - & - \\
\hline Sorensen (31) & 2012 & 65 & ВТТ: 100\% & NR & $23.10 \%$ & - & - & - & - & - & - & - & $10.70 \%$ & - \\
\hline Yuan (32) & 2012 & 133 & BTT: $51 \%$; DT: $30 \%$; other $19 \%$ & Mean 303 days support & - & - & - & - & - & - & - & - & $21.50 \%$ & - \\
\hline Dell'Aquila (33) & 2013 & 50 & ВТТ: 100\% & 30.3 & $36 \%$ & $26 \%$ & $26 \%$ & $4 \%$ & - & $14 \%$ & $28 \%$ & - & - & - \\
\hline Kutty (35) & 2013 & 29 & BTT: $41 \%$; other: $59 \%$ & Mean 327 days support & - & - & - & - & - & - & - & - & $13.80 \%$ & - \\
\hline Lok (36) & 2013 & 85 & ВТТ: 100\% & 109.1 & - & $5 \%$ & $11 \%$ & $5 \%$ & - & $14 \%$ & $27 \%$ & $5 \%$ & $4.70 \%$ & $27.10 \%$ \\
\hline Meyer (37) & 2013 & 115 & BTT: 92\%; DT: 8\% & NR & $45.20 \%$ & - & - & - & $4.30 \%$ & $27.80 \%$ & - & $0.9 \% \%$ & - & - \\
\hline Mulloy (38) & 2013 & 50 & NR (BTT and DT) & Mean 273 days follow up & $22 \%$ & $22 \%$ & $8 \%$ & - & - & - & $14 \%$ & $2 \%$ & - & - \\
\hline Saeed (39) & 2013 & 21 & $\mathrm{NR}$ (BTT and DT) & 17 & - & - & - & - & - & - & - & - & - & - \\
\hline Sakaguchi (40) & 2013 & 23 & ВT: $100 \%$ & 35 & $13 \%$ & - & $4.30 \%$ & $13 \%$ & - & $13 \%$ & - & $21.70 \%$ & $21.70 \%$ & - \\
\hline Sabashnikov (42) & 2014 & 139 & ВТТ: 100\% & Mean 514 days support & - & - & $9 \%$ & $14 \%$ & - & $25 \%$ & $10 \%$ & $1 \%$ & - & - \\
\hline Takeda (43) & 2014 & 140 & BTT: 82\%; DT: 18\% & Mean 438 days support & - & - & - & - & - & - & - & - & $3.60 \%$ & - \\
\hline
\end{tabular}




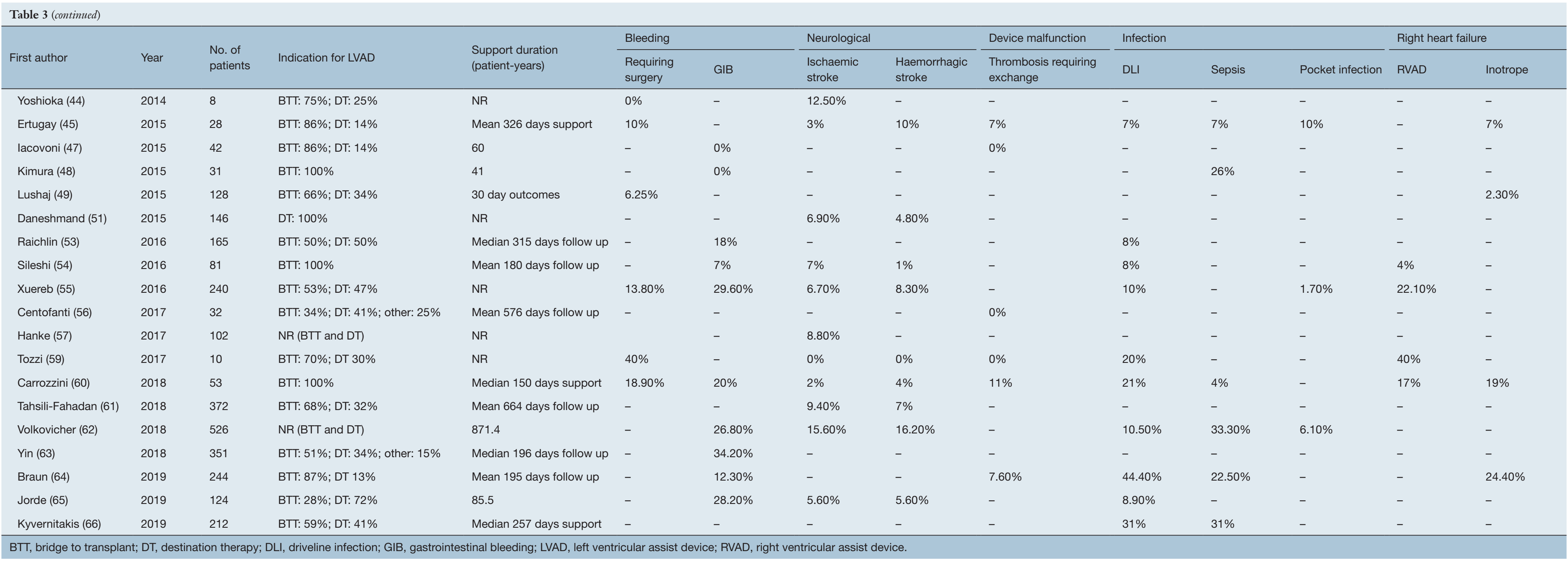




\begin{tabular}{|c|c|c|c|c|c|c|c|c|c|c|c|c|c|c|}
\hline \multirow[b]{2}{*}{ First author } & \multirow[b]{2}{*}{ Year } & \multirow{2}{*}{$\begin{array}{l}\text { No. of } \\
\text { patients }\end{array}$} & \multirow[b]{2}{*}{ Indication for LVAD } & \multirow[b]{2}{*}{ Support duration (patient-years) } & \multicolumn{2}{|l|}{ Bleeding } & \multicolumn{2}{|c|}{ Neurological } & \multirow{2}{*}{$\begin{array}{l}\text { Device malfunction } \\
\text { Thrombosis requiring } \\
\text { exchange }\end{array}$} & \multicolumn{3}{|c|}{ Infection } & \multicolumn{2}{|c|}{ Right heart failure } \\
\hline & & & & & $\begin{array}{l}\text { Requiring } \\
\text { surgery }\end{array}$ & GIB & $\begin{array}{l}\text { Ischaemic } \\
\text { stroke }\end{array}$ & $\begin{array}{l}\text { Haemorrhagic } \\
\text { stroke }\end{array}$ & & DLI & Sepsis & Pocket infection & RVAD & Inotrope \\
\hline \multicolumn{15}{|c|}{ Industry-funded trials and associated registries } \\
\hline Bogaev (11) & 2011 & 465 & ВТТ: $100 \%$ & 431.2 & 0.26 & - & 0.05 & 0.05 & - & - & 0.31 & - & - & - \\
\hline Strueber (12) & 2011 & 50 & ВТТ: $100 \%$ & 47.8 & 0.23 & - & 0.04 & 0.08 & 0.02 & 0.20 & 0.10 & - & 0.06 & 0.06 \\
\hline Park (13) & 2012 & 414 & DT: $100 \%$ & 709 & 0.17 & - & 0.05 & 0.04 & 0.01 & 0.27 & 0.30 & 0.06 & 0.03 & 0.14 \\
\hline Najjar (15) & 2014 & 382 & ВТТ: $100 \%$ & 406.6 & 0.16 & 0.27 & 0.06 & 0.08 & 0.04 & 0.25 & 0.23 & - & 0.04 & 0.32 \\
\hline Netuka (17) & 2017 & 101 & ВTT: 82\%; DT 18\% & 205.96 & - & - & 0.02 & 0.02 & - & - & - & - & - & - \\
\hline Rogers (18) & 2017 & 446 & DT: $100 \%$ & 613.96 & 0.13 & 0.52 & 0.13 & 0.09 & - & 1.27 & 0.18 & - & 0.02 & 0.27 \\
\hline Schmitto (6) & 2019 & 50 & BTT: 54\%; DT: 46\% & 77.4 & 0.14 & 0.18 & 0.10 & 0.05 & 0.00 & 0.22 & 0.14 & - & 0.03 & 0.09 \\
\hline \multicolumn{15}{|c|}{ Single- and multi-center case series } \\
\hline Strüber (21) & 2008 & 101 & BTT: 69\%; DT: 31\% & 44.59 & - & - & 0.07 & 0.05 & - & 0.37 & - & - & - & - \\
\hline Wieselthaler (24) & 2010 & 23 & ВТТ: $100 \%$ & 19 & 0.16 & 0.16 & 0.05 & 0.05 & - & 0.42 & 0.16 & - & 0.05 & - \\
\hline Menon (28) & 2012 & 40 & ВТ: 63\%; DT: 23\%; other: 15\% & 28.95 & 0.24 & 0.10 & 0.07 & - & - & - & - & - & - & - \\
\hline Dell'Aquila (33) & 2013 & 50 & ВТТ: 100\% & 30.3 & 0.59 & 0.43 & 0.43 & 0.07 & - & 0.23 & 0.46 & - & - & - \\
\hline Lok (36) & 2013 & 85 & ВТТ: $100 \%$ & 109.1 & - & 0.05 & 0.08 & 0.04 & - & 0.13 & 0.31 & 0.04 & 0.04 & 0.21 \\
\hline Meyer (37) & 2013 & 115 & ВTT: 92\%; DT: 8\% & NR & 0.39 & - & - & - & 0.04 & 0.24 & - & - & - & - \\
\hline Hata (46) & 2015 & 32 & ВТТ: $100 \%$ & 33.9 & 0.32 & - & - & - & - & - & - & - & - & - \\
\hline lacovoni (47) & 2015 & 42 & BTT: 86\%; DT: 14\% & 60 & - & 0.00 & - & - & 0.00 & 0.33 & 0.07 & - & - & - \\
\hline John (52) & 2016 & 267 & ВТТ: 79\%; DT: 21\% & 479.01 & - & 0.14 & - & - & - & 0.15 & - & - & - & - \\
\hline Volkovicher (62) & 2018 & 526 & NR (BTT and DT) & 871.4 & - & 0.25 & 0.11 & 0.11 & - & 0.13 & 0.41 & 0.07 & - & - \\
\hline Jorde (65) & 2019 & 124 & BTT: 28\%; DT: 72\% & 84.7 & - & 0.71 & 0.08 & 0.08 & - & 0.18 & - & - & - & - \\
\hline
\end{tabular}




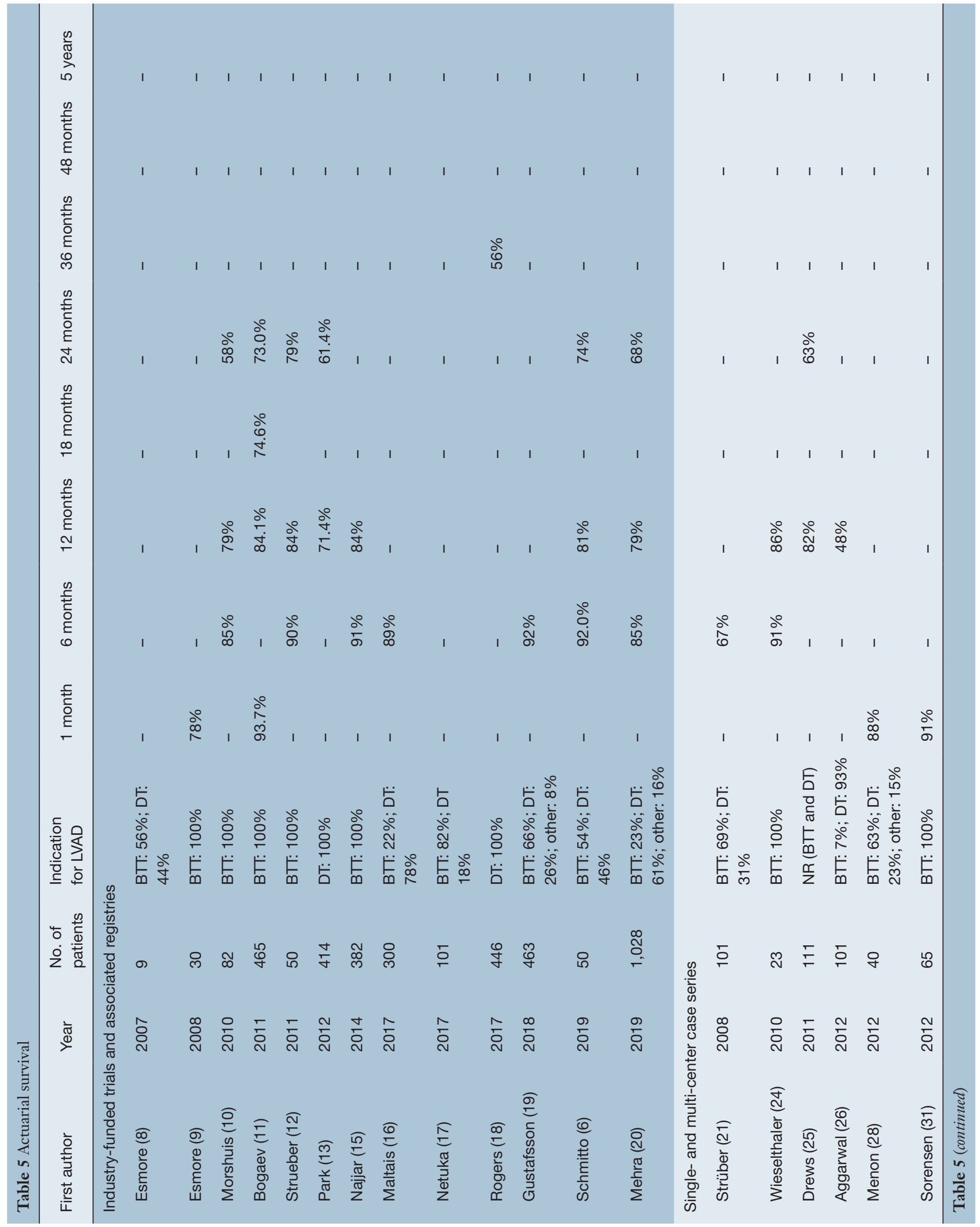




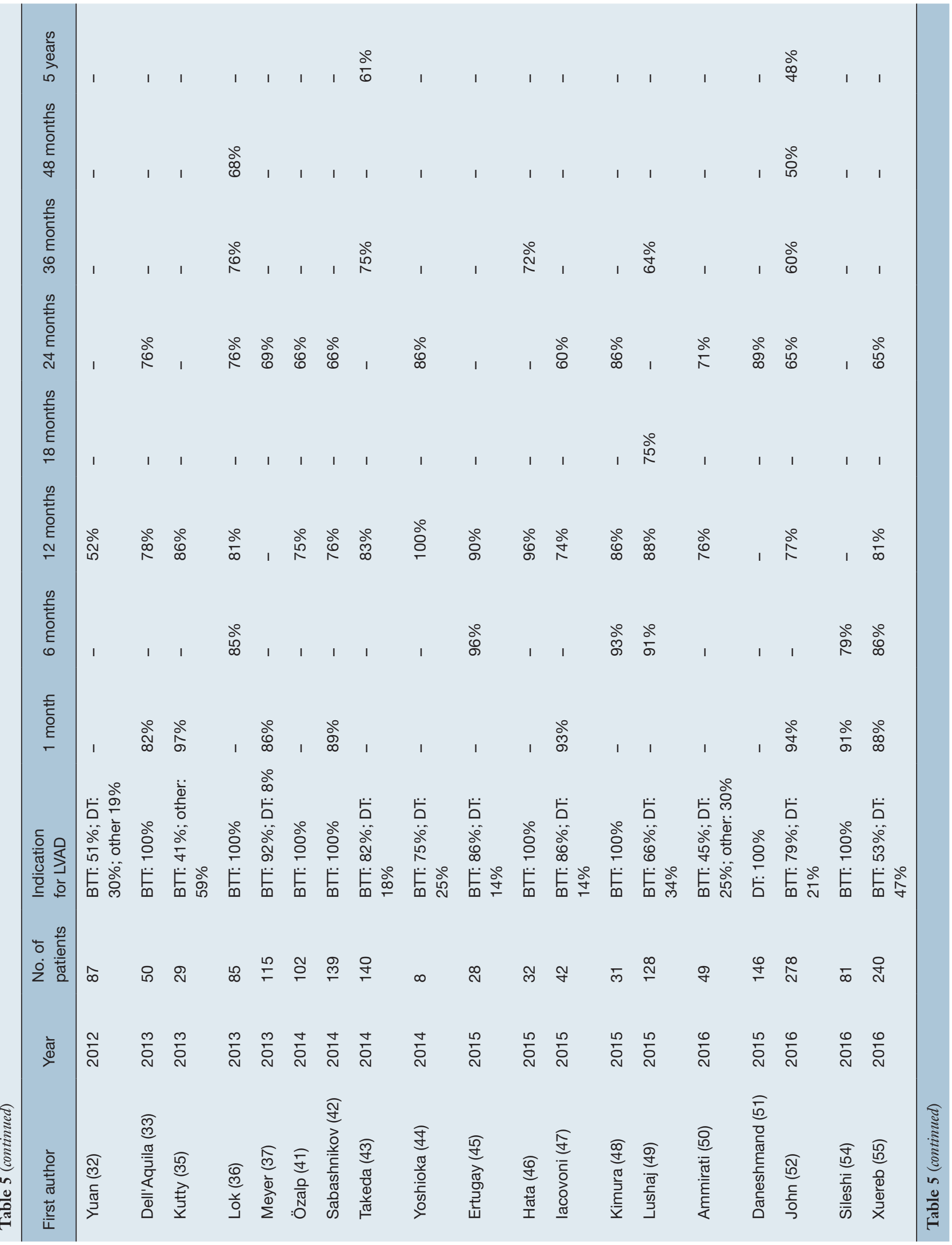




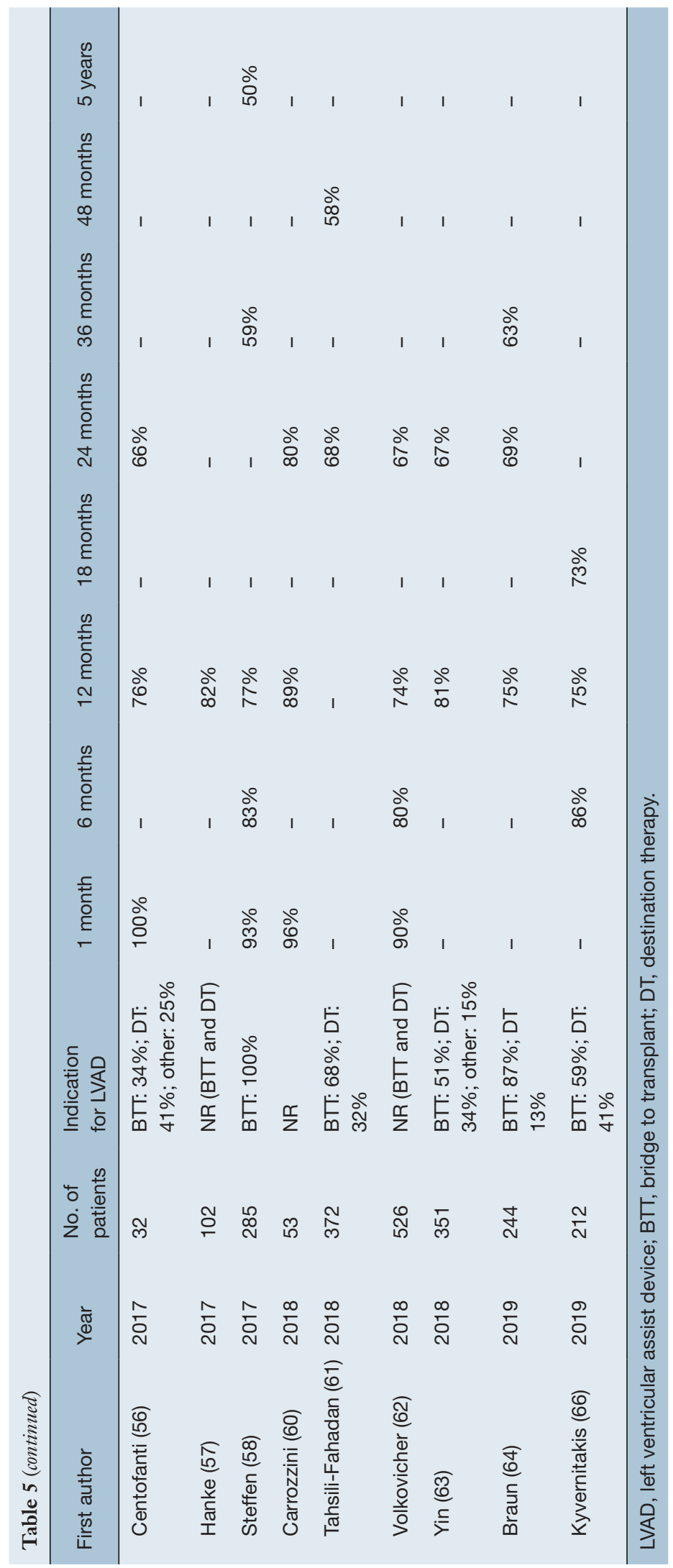

(C) Annals of Cardiothoracic Surgery. All rights reserved. Ann Cardiothorac Surg 2021;10(2):186-208 I http://dx.doi.org/10.21037/acs-2021-cfmcs-35 
center case series (Table 3) reported the incidence of pump thrombosis requiring device exchange. Three studies reported no patients requiring device replacement for pump thrombosis, while the remaining eleven studies reported an incidence of between $2 \%$ and $11 \%$ of LVAD recipients. This is still significantly less than the nearly $50 \%$ of patients implanted with the first-generation HeartMate XVE who experienced device exchange due to malfunction or infection at 18 months (73). Expressed as the number of events per patient-year, this was a relatively uncommon occurrence (less than 0.04 events per patient-year).

\section{Right beart failure (RHF)}

RHF has been associated with poorer outcomes and increased short-term mortality (74). In this review ten industry-funded studies and sixteen single- and multi-center case series (Table 3) reported on the incidence of RHF either requiring prolonged inotropic support or placement of a right ventricular assist device (RVAD). RHF requiring prolonged inotropic support was present in $2 \%$ to $32 \%$ of patients, while RHF requiring placement of an RVAD was reported in $2.9 \%$ to $40 \%$ of patients. This is significantly higher than the $19.7 \%$ and $6.2 \%$ of patients requiring prolonged inotropic support and RVAD implantation reported from the HMII Trial and Continued Access Protocol registry (11). Sensitivity analysis was performed where studies with less than 100 participants were excluded, following which the rates of prolonged inotropic support and requirement for RVAD placement were $8 \%$ to $32 \%$ and $3 \%$ to $7 \%$ respectively in the industry-funded trials. Similar sensitivity analysis performed looking at the singleand multi-center case series showed a requirement for prolonged inotropic support and RVAD placement of $2 \%$ to $24 \%$ and $4 \%$ to $22 \%$, respectively. This sensitivity analysis shows that the incidence of RVAD placement was overestimated in several small studies but is generally higher than the rates reported in the large clinical trials, perhaps reflecting the influence of patient selection through the use of inclusion and exclusion criteria.

\section{Health related quality of life (HRQoL)}

Commonly used measures of health-related quality of life were the Kansas City Cardiomyopathy Questionnaire (KCCQ), and Minnesota Living with Heart Failure Questionnaire (MLHFQ). Overall, quality of life was infrequently assessed and reported exclusively by industry-funded trials. The KCCQ is a self-administered questionnaire that examines physical function, social function, symptoms and quality of life. An overall summary score can be derived from the various domains, measured on a scale from $0-100$, in which higher scores reflect better health status (75). Five industry-funded trials $(11,12-14,20)$ assessed HRQoL after LVAD implantation using the KCCQ. Baseline scores were similar, ranging from 28 to 40 , and significant differences were detected by six months (63 to 72). Two studies continued assessing KCCQ out to 24 months and showed that the improvements achieved at six months were maintained 12- and 24-month post LVADimplantation (Table 6). These findings were consistent with those reported by Kormos and colleagues (69) in a large registry report that included over 18,000 patients who received cf-LVAD for BTT and DT.

The MLHFQ questionnaire assesses two domains, physical and emotional, and provides a summary score on a scale from 0-105 (76). However, unlike the KCCQ, higher scores on the MLHFQ correlate with more significant impairment in health-related quality of life. Two industryfunded trials $(11,13)$ examined quality of life using the MLHFQ and demonstrated significant improvements by six months that were maintained through to 12- and 24-month post LVAD implantation.

\section{Functional outcomes}

Commonly used tools for the assessment of function after LVAD implantation were the New York Heart Association (NYHA) status and the 6-minute walk test (6MWT). Four industry-funded trials $(6,11,13,20)$ assessed NYHA status at baseline and post-LVAD implantation. Essentially all patients were classified as NYHA III or IV at baseline, with $79-85 \%$ improving to NYHA I-II at six months and no evidence of deterioration in status at 24 months. Three industry-funded trials $(11,14,20)$ assessed $6 \mathrm{MWT}$ at baseline and post-LVAD implantation. Many patients were unable to participate in the $6 \mathrm{MWT}$ at baseline. Significant improvements in distances walked were evident by six months and maintained through to 24 months post LVADimplantation.

\section{Discussion}

LVAD therapy offers patients with ESHF the potential for improved survival, HRQoL and functional status compared with medical therapy alone. However, there are several risks associated with these devices that can limit survival 
Table 6 Functional and quality of life outcomes

\begin{tabular}{|c|c|c|c|c|c|c|c|c|c|c|c|c|c|c|c|c|c|c|c|}
\hline \multirow[b]{2}{*}{ First author } & \multirow[b]{2}{*}{ Year } & \multirow{2}{*}{$\begin{array}{l}\text { No. of } \\
\text { patients }\end{array}$} & \multirow{2}{*}{$\begin{array}{l}\text { Indication for } \\
\text { LVAD (\%) }\end{array}$} & \multicolumn{4}{|c|}{ NYHA status } & \multicolumn{4}{|l|}{$6 \mathrm{MWT}$} & \multicolumn{4}{|l|}{ MLHFQ } & \multicolumn{4}{|l|}{$\mathrm{KCCQ}$} \\
\hline & & & & $\begin{array}{l}\text { NYHA I-II } \\
\text { baseline }\end{array}$ & $\begin{array}{l}\text { NYHA I-II } \\
6 \text { months }\end{array}$ & $\begin{array}{l}\text { NYHA I-II } \\
12 \text { months }\end{array}$ & $\begin{array}{l}\text { NYHA I-II } \\
24 \text { months }\end{array}$ & $\begin{array}{l}6 \mathrm{MWT} \\
\text { baseline ( } \mathrm{m})\end{array}$ & $\begin{array}{l}6 \mathrm{MWT} \\
6 \text { months (m) }\end{array}$ & $\begin{array}{l}6 \mathrm{MWT} \\
12 \text { months (m) }\end{array}$ & $\begin{array}{l}6 \mathrm{MWT} \\
24 \text { months }(\mathrm{m})\end{array}$ & $\begin{array}{l}\text { MLHFQ } \\
\text { baseline (mean) }\end{array}$ & $\begin{array}{l}\text { MLHFQ } \\
6 \text { months (mean) }\end{array}$ & $\begin{array}{l}\text { MLHFQ } \\
12 \text { months (mean) }\end{array}$ & $\begin{array}{l}\text { MLHFQ } \\
12 \text { months (mean) }\end{array}$ & $\begin{array}{l}\mathrm{KCCQ} \\
\text { baseline }\end{array}$ & $\begin{array}{l}\mathrm{KCCQ} \\
6 \text { months }\end{array}$ & $\begin{array}{l}\text { KCCQ } \\
12 \text { months }\end{array}$ & $\begin{array}{l}\text { KCCQ } \\
24 \text { months }\end{array}$ \\
\hline \multicolumn{20}{|c|}{ Industry-funded trials and associated registries } \\
\hline Bogaev (11) & 2011 & 465 & ВТT: $100 \%$ & 0 & $85 \%$ & - & - & 225 & 333 & - & - & 72.5 & 36.1 & - & - & 29 & 67 & - & - \\
\hline Strueber (12) & 2011 & 50 & ВTT: $100 \%$ & - & - & - & - & - & - & - & - & - & - & - & - & 30 & 72 & - & - \\
\hline Park (13) & 2012 & 414 & DT: $100 \%$ & 0 & $81 \%$ & $77 \%$ & $80 \%$ & - & - & - & - & 75 & 33 & 32 & 31 & 28 & 68 & 69 & 70 \\
\hline Slaughter (14) & 2013 & 332 & ВТТ: $100 \%$ & - & - & - & - & 75 & 255 & - & - & - & - & - & - & 37 & 68 & - & - \\
\hline Schmitto (6) & 2019 & 50 & $\begin{array}{l}\text { BTT: } 54 \% ; \text { DT: } \\
46 \%\end{array}$ & 0 & - & - & $88 \%$ & - & - & - & - & - & - & - & - & - & - & - & - \\
\hline Mehra (20) & 2019 & 1,028 & $\begin{array}{l}\text { BTT: 23\%; DT: } \\
\text { 61\%; other: 16\% }\end{array}$ & 0 & $79 \%$ & $81 \%$ & $79 \%$ & 132 & 318 & 329 & 340 & - & - & - & - & 40 & 70 & 68 & 69 \\
\hline \multicolumn{20}{|l|}{ Registry report } \\
\hline Kormos (69) & 2019 & 18,539 & BTT: $67 \%$ DT: 43\% & - & - & - & - & - & - & - & - & - & - & - & - & 34.5 & 67 & 66.8 & 65.9 \\
\hline
\end{tabular}


and long-term use if not appropriately managed. The most commonly reported major events after LVAD implantation are bleeding requiring surgery, GIB, neurological events, pump thrombosis, infection and right ventricular failure. Definitions for these adverse events varied between studies included in this review, but for the majority the INTERMACS definitions were used. Significant variances were seen in the reported incidence of adverse events between studies due mainly to the differing follow-up periods, as many of the reported adverse outcomes are proportional to the time at risk. The significance of these adverse events becomes pronounced when we consider that more patients are receiving LVAD with a DT indication and the cumulative risk of GIB, infection and stroke increases proportionally with the duration of support (77).

Previous reviews have examined associations between LVAD and single adverse events, compared outcomes following LVAD versus OHT, and examined outcomes following OHT in patients bridged with LVAD against those who were not. The last review that attempted to broadly examine outcomes after cf-LVAD was performed by McIlvennan et al. (78) in 2014, and there has subsequently been a large volume of relevant publications in the interceding six years. Another considerable change over this time has been the expansion of devices approved for use, both as BTT and DT, a shift towards LVAD as DT (at least in the USA) and the development of new thirdgeneration centrifugal flow devices. For these reasons we believed there was need for an updated systematic review reporting survival and adverse outcomes after cf-LVAD implantation. More specific analysis of individual devices, or even centrifugal- versus axial-flow LVADs is not within the scope of this review and should be the subject of their own, more specific systematic review.

The use of cf-LVAD has been shown to have similar two-year survival to that of OHT $(12,79)$ and offers the potential to improve quality of life and functional status of patients with ESHF. Survival after cf-LVAD implantation in this review was generally good, with the results of industryfunded trials similar to those published by International Society of Heart and Lung Transplantation (ISHLT) in the IMACS third annual report. Single- and multi-center registries published some of the poorer survival outcomes, and generally these results were from small studies with a large proportion of DT recipients. Other possible causes for this observed difference include less selective inclusion criteria, potentially sicker or more comorbid patients, and the fact that some of the small single-centre case series were publications of a unit's initial experience with a particular device.

Infection has always been an important cause of morbidity and mortality in patients supported with LVAD. In the pivotal REMATCH trial (3) the survival benefit of pfLVAD failed to extend beyond the first year of support and the predominant cause of death in the treatment arm was sepsis, responsible for $41 \%$ of deaths. Since the introduction of cf-LVAD devices, multiple studies have demonstrated a reduction in DLI and sepsis and near eradication of pump pocket infections (80-83). Recognition of this potentially fatal complication has led to the early identification and treatment of DLI and subsequently reduced the morbidity and mortality associated with device related infections.

Bleeding has always been a frequent adverse event following LVAD implantation, but the introduction of cfLVAD devices has seen a significant change in the type of bleeding experienced by patients. While the pulsatile devices were associated with frequent early postoperative bleeding the newer devices are associated with higher rates of late bleeding, particularly GIB $(4,70,83,84-86)$. The main differences between the two devices are the requirement for the formation of a large pump pocket for the pf-LVAD, and the requirement for anticoagulation with the cf-LVAD to prevent pump thrombosis. While the first difference may explain the reduced rates of early postoperative bleeding, anticoagulation alone does not explain the increased risk of late bleeding. Observed rates of late bleeding in cf-LVAD patients of 63 GIB events/100 patient-years significantly exceeds the documented rates of bleeding in patients receiving anticoagulation for mechanical valves (4.6 bleeding events/100 patient-years) $(87,88)$. Additionally, several studies have observed bleeding in the absence of supratherapeutic INR and no significant difference in the average INR observed at the time of bleeding compared with those without bleeding $(70,84,87,89)$. These two observations and the fact that removal of the device for cardiac recovery or heart transplantation results in an abatement of GIB (84) suggest that there are specific factors associated with cf-LVADs that are responsible for the development of a bleeding diathesis. Several proposed mechanisms centre around the development of an acquired von Willebrand syndrome and the loss of pulsatile flow (71,90-92). An acquired von Willebrand syndrome develops in all patients on cf-LVAD support as demonstrated by the decrease in or absence of high molecular weight (HMW) von Willebrand factor (vWF) multimers $(26,71)$. The cause of this is two-fold: firstly, these HMW multimers 
rely upon pulsatile flow for their release from endothelial cells; secondly, the high shear stress created by axialflow impellers results in the destruction of the largest multimers (26). These HMW vWF multimers are essential for platelet activation and aggregation at sites of bleeding, particularly at sites of high shear stress such as arteriovenous malformations (AVMs) (93-96). The second factor thought to contribute to the bleeding diathesis is the presence of non-pulsatile flow, which may actually increase the development of gastrointestinal angiodysplasia. Investigators have proposed that narrow pulse pressure may increase intraluminal pressure and lead to dilatation of the sub-mucosal venous plexus, predisposing to angiodysplasia, AVM formation and eventually bleeding $(86,97,98)$ There are two observations supporting the idea that the loss of pulsatile flow contributes to the development of GIB: firstly, the observation that the absence of aortic valve opening was associated with over a four-fold increase in the risk of non-surgical bleeding (70), and secondly, that reduction in flow rates to allow aortic valve opening tends to restore pulsatility and ameliorates GIB $(87,98)$.

Newer, third generation LVADs utilise a magnetically levitated centrifugal impeller to generate flow. The small housing and contactless bearing reduce blood-biomaterial interface, reduce heat generation, and permit regular alterations in pump speed to generate a pseudo-pulsatile flow pattern. Early results appear to confirm that these improvements have resulted in a reduced risk of $\operatorname{GIB}(20,99)$. However, as third generation devices are relatively new, results are mostly from industry-funded trials and long-term follow-up is lacking in comparison to axial-flow devices.

An observation of this review was that industryfunded trials tended to have less variance in the reported proportions of patients experiencing adverse outcomes compared with those results reported from single- and multi-center case series. This is likely due to a highly selected patient cohort with more stringent inclusion and exclusion criteria, and a more uniform definition of outcomes. Another potential contributing factor may be the presence of small sample size bias in the case series; but without assessing the data statistically for the presence of such bias, we cannot ignore the fact that the difference may also represent true outcomes from a less restrictive cohort. Thus, we must be careful not to apply the findings from industry-funded trials to all patients, particularly those who may be older, carry more comorbidities or are receiving the device for DT indications, without some thought as to the applicability of those results to the individual.
This review observed a trend towards higher rates of adverse events reported in DT trials than BTT trials as has been previously reported (100). It is difficult to know whether this is truly the case as there was a paucity of trials assessing outcomes for DT-only cohorts. Whether this observation represents the outcomes of a generally older and sicker patient cohort or is due to prolonged duration of cf-LVAD support is unclear and beyond the scope of this review but should be kept in mind when interpreting the data presented in the above tables.

\section{Limitations}

There are several limitations to this review that need addressing. Firstly, the authors endeavored to produce a comprehensive systematic review of cf-LVAD outcomes. The broad inclusion criteria were designed to capture as many studies reporting survival, adverse events, quality of life and functional status as possible. The downside to such an approach is the inclusion of a large number of studies, with significant overlap and a large degree of heterogeneity between studies in regard to patient inclusion criteria, baseline characteristics and duration of follow up. The lack of standard definitions, non-uniform reporting of outcome event rates and varying durations of follow up limited the ability to pool data and meant that meta-analysis was not appropriate. Secondly, while there were a few randomized trials and prospective cohort studies, the way the data were extracted from the studies meant that each study was essentially treated as a case-series, precluding any assessment of the quality of the evidence as case-series are considered to be of poor quality and there are no validated tools for this study design. Additionally, the mixing of BTT and DT patients in the majority of single- and multi-center case series made a separate comparison of BTT and DT outcomes difficult. The resulting descriptive analysis is, therefore, not specific to patients undergoing implantation for either indication but a general overview of outcomes following implantation.

\section{Conclusions}

There is an ever-increasing amount of data pertaining to survival and long-term outcomes after LVAD implantation. Studies have consistently shown an increase in survival, quality of life and functional status following cf-LVAD implantation, but these devices remain associated with significant morbidity and mortality in the long-term. 
While short-term survival remains comparable with OHT, complications such as bleeding, infection and stroke have been associated with poorer long-term survival, and the cumulative risk of experiencing these adverse events is directly proportional to the duration of support. With more people receiving LVAD support and no commensurate increase in donor organs, these complications are going to be a growing cause of morbidity, mortality and rehospitalization. While there is a growing understanding of why these complications occur there remains a need for the development of management strategies to effectively minimize these risks. There remains a need for large studies of high quality to examine the long-term outcomes of cfLVAD for DT indication and for investigating management strategies to minimize the incidence of complications. The findings of this review suggest that despite the increasing occurrence of adverse events over the long-term, patients supported with cf-LVAD continue to experience an improved quality of life and functional status that does not decline with prolonged support. This is testament to the usefulness of cf-LVAD therapy in the treatment of end stage heart failure, an otherwise highly morbid and fatal medical condition.

\section{Acknowledgments}

Funding: Principal investigator NM received financial support from the Baird Institute in the form of a scholarship.

\section{Footnote}

Conflicts of Interest: The authors have no conflicts of interest to declare.

Open Access Statement: This is an Open Access article distributed in accordance with the Creative Commons Attribution-NonCommercial-NoDerivs 4.0 International License (CC BY-NC-ND 4.0), which permits the noncommercial replication and distribution of the article with the strict proviso that no changes or edits are made and the original work is properly cited (including links to both the formal publication through the relevant DOI and the license). See: https://creativecommons.org/licenses/by-nc-nd/4.0/.

\section{References}

1. Lippi G, Sanchis-Gomar F. Global epidemiology and future trends of heart failure. AME Med J 2020;5:15.

2. Stehlik J, Kobashigawa J, Hunt SA, et al. Honoring 50 Years of Clinical Heart Transplantation in Circulation: In-Depth State-of-the-Art Review. Circulation 2018;137:71-87.

3. Rose EA, Gelijns AC, Moskowitz AJ, et al. Long-term use of a left ventricular assist device for end-stage heart failure. N Engl J Med 2001;345:1435-43.

4. Slaughter MS, Rogers JG, Milano CA, et al. Advanced heart failure treated with continuous-flow left ventricular assist device. N Engl J Med 2009;361:2241-51.

5. Milano CA, Rogers JG, Tatooles AJ, et al. HVAD: The ENDURANCE Supplemental Trial. JACC Heart Fail 2018;6:792-802.

6. Schmitto JD, Pya Y, Zimpfer D, et al. Long-term evaluation of a fully magnetically levitated circulatory support device for advanced heart failure-two-year results from the HeartMate 3 CE Mark Study. Eur J Heart Fail 2019;21:90-7.

7. Moher D, Liberati A, Tetzlaff J, et al. Preferred reporting items for systematic reviews and meta-analyses: the PRISMA statement. PLoS Med 2009;6:e1000097.

8. Esmore D, Spratt P, Larbalestier R, et al. VentrAssist left ventricular assist device: clinical trial results and Clinical Development Plan update. Eur J Cardiothorac Surg 2007;32:735-44.

9. Esmore DS, Kaye D, Salamonsen R, et al. Initial clinical experience with the VentrAssist left ventricular assist device: the pilot trial. J Heart Lung Transplant 2008;27:479-85.

10. Morshuis M, Schoenbrodt M, Nojiri C, et al. DuraHeart magnetically levitated centrifugal left ventricular assist system for advanced heart failure patients. Expert Rev Med Devices 2010;7:173-83.

11. Bogaev RC, Pamboukian SV, Moore SA, et al. Comparison of outcomes in women versus men using a continuous-flow left ventricular assist device as a bridge to transplantation. J Heart Lung Transplant 2011;30:515-22.

12. Strueber M, O'Driscoll G, Jansz P, et al. Multicenter evaluation of an intrapericardial left ventricular assist system. J Am Coll Cardiol 2011;57:1375-82.

13. Park SJ, Milano CA, Tatooles AJ, et al. Outcomes in advanced heart failure patients with left ventricular assist devices for destination therapy. Circ Heart Fail 2012;5:241-8.

14. Slaughter MS, Pagani FD, McGee EC, et al. HeartWare ventricular assist system for bridge to transplant: combined results of the bridge to transplant and continued access 
protocol trial. J Heart Lung Transplant 2013;32:675-83.

15. Najjar SS, Slaughter MS, Pagani FD, et al. An analysis of pump thrombus events in patients in the HeartWare ADVANCE bridge to transplant and continued access protocol trial. J Heart Lung Transplant 2014;33:23-34.

16. Maltais S, Kilic A, Nathan S, et al. PREVENtion of HeartMate II Pump Thrombosis Through Clinical Management: The PREVENT multi-center study. J Heart Lung Transplant 2017;36:1-12.

17. Netuka I, Litzler PY, Berchtold-Herz M, et al. Outcomes in HeartMate II Patients With No Antiplatelet Therapy: 2-Year Results From the European TRACE Study. Ann Thorac Surg 2017;103:1262-8.

18. Rogers JG, Pagani FD, Tatooles AJ, et al. Intrapericardial Left Ventricular Assist Device for Advanced Heart Failure. N Engl J Med 2017;376:451-60.

19. Gustafsson F, Shaw S, Lavee J, et al. Six-month outcomes after treatment of advanced heart failure with a full magnetically levitated continuous flow left ventricular assist device: report from the ELEVATE registry. Eur Heart J 2018;39:3454-60.

20. Mehra MR, Uriel N, Naka Y, et al. A Fully Magnetically Levitated Left Ventricular Assist Device - Final Report. N Engl J Med 2019;380:1618-27.

21. Strüber M, Sander K, Lahpor J, et al. HeartMate II left ventricular assist device; early European experience. Eur J Cardiothorac Surg 2008;34:289-94.

22. Loforte A, Montalto A, Ranocchi F, et al. Long-term mechanical support with the HeartMate II LVAS. Transplant Proc 2009;41:1357-9.

23. Sandner SE, Zimpfer D, Zrunek P, et al. Age and outcome after continuous-flow left ventricular assist device implantation as bridge to transplantation. J Heart Lung Transplant 2009;28:367-72.

24. Wieselthaler GM, O Driscoll G, Jansz P, et al. Initial clinical experience with a novel left ventricular assist device with a magnetically levitated rotor in a multi-institutional trial. J Heart Lung Transplant 2010;29:1218-25.

25. Drews T, Dandel M, Krabatsch T, et al. Long-term mechanical circulatory support in 198 patients: largest single-center experience worldwide. ASAIO J 2011;57:9-16.

26. Aggarwal A, Pant R, Kumar S, et al. Incidence and management of gastrointestinal bleeding with continuous flow assist devices. Ann Thorac Surg 2012;93:1534-40.

27. Brenyo A, Rao M, Koneru S, et al. Risk of mortality for ventricular arrhythmia in ambulatory LVAD patients. J Cardiovasc Electrophysiol 2012;23:515-20.

28. Menon AK, Götzenich A, Sassmannshausen H, et al.
Low stroke rate and few thrombo-embolic events after HeartMate II implantation under mild anticoagulation. Eur J Cardiothorac Surg 2012;42:319-23; discussion 323.

29. Ozbaran M, Yagdi T, Engin C, et al. New circulatory support system: heartware. Transplant Proc 2012;44:1726-8.

30. Raasch H, Jensen BC, Chang PP, et al. Epidemiology, management, and outcomes of sustained ventricular arrhythmias after continuous-flow left ventricular assist device implantation. Am Heart J 2012;164:373-8.

31. Sorensen EN, Pierson RN 3rd, Feller ED, et al. University of Maryland surgical experience with the Jarvik 2000 axial flow ventricular assist device. Ann Thorac Surg 2012;93:133-40.

32. Yuan N, Arnaoutakis GJ, George TJ, et al. The spectrum of complications following left ventricular assist device placement. J Card Surg 2012;27:630-8.

33. Dell'Aquila AM, Schneider SR, Schlarb D, et al. Initial clinical experience with the HeartWare left ventricular assist system: a single-center report. Ann Thorac Surg 2013;95:170-7.

34. Forest SJ, Bello R, Friedmann P, et al. Readmissions after ventricular assist device: etiologies, patterns, and days out of hospital. Ann Thorac Surg 2013;95:1276-81.

35. Kutty RS, Parameshwar J, Lewis C, et al. Use of centrifugal left ventricular assist device as a bridge to candidacy in severe heart failure with secondary pulmonary hypertension. Eur J Cardiothorac Surg 2013;43:1237-42.

36. Lok SI, Martina JR, Hesselink T, et al. Single-centre experience of 85 patients with a continuous-flow left ventricular assist device: clinical practice and outcome after extended support. Eur J Cardiothorac Surg 2013;44:e233-8.

37. Meyer AL, Malehsa D, Bara C, et al. Implantation of rotary blood pumps into 115 patients: a single-centre experience. Eur J Cardiothorac Surg 2013;43:1233-6.

38. Mulloy DP, Bhamidipati CM, Stone ML, et al. Cryoablation during left ventricular assist device implantation reduces postoperative ventricular tachyarrhythmias. J Thorac Cardiovasc Surg 2013;145:1207-13.

39. Saeed D, Arusoglu L, Gazzoli F, et al. Results of the European clinical trial of Arrow CorAide left ventricular assist system. Artif Organs 2013;37:121-7.

40. Sakaguchi T, Matsumiya G, Yoshioka D, et al. DuraHeart ${ }^{\mathrm{TM}}$ magnetically levitated left ventricular assist device: Osaka University experience. Circ J 2013;77:1736-41. 
41. Özalp F, Bhagra S, Bhagra C, et al. Four-year outcomes with third-generation centrifugal left ventricular assist devices in an era of restricted transplantation. Eur J Cardiothorac Surg 2014;46:e35-40.

42. Sabashnikov A, Mohite PN, Weymann A, et al F. Outcomes after implantation of 139 full-support continuous-flow left ventricular assist devices as a bridge to transplantation. Eur J Cardiothorac Surg 2014;46:e59-66.

43. Takeda K, Takayama H, Kalesan B, et al. Long-term outcome of patients on continuous-flow left ventricular assist device support. J Thorac Cardiovasc Surg 2014;148:1606-14.

44. Yoshioka D, Matsumiya G, Toda K, et al. Clinical results with Jarvik 2000 axial flow left ventricular assist device: Osaka University Experience. J Artif Organs 2014;17:308-14.

45. Ertugay S, Engin C, Nalbantgil S, et al. Postoperative Outcomes of the Largest HeartMate-II Experience in Turkey. Transplant Proc 2015;47:1499-502.

46. Hata H, Fujita T, Shimahara Y, et al. Early and mid-term outcomes of left ventricular assist device implantation and future prospects. Gen Thorac Cardiovasc Surg 2015;63:557-64.

47. Iacovoni A, Centofanti P, Attisani M, et al. Low incidence of gastrointestinal bleeding and pump thrombosis in patients receiving the INCOR LVAD system in the longterm follow-up. Int J Artif Organs 2015;38:542-7.

48. Kimura M, Kinoshita O, Nawata K, et al. Midterm outcome of implantable left ventricular assist devices as a bridge to transplantation: Single-center experience in Japan. J Cardiol 2015;65:383-9.

49. Lushaj EB, Badami A, Osaki S, et al. Impact of age on outcomes following continuous-flow left ventricular assist device implantation. Interact Cardiovasc Thorac Surg 2015;20:743-8.

50. Ammirati E, Oliva FG, Colombo T, et al. Mid-term survival after continuous-flow left ventricular assist device versus heart transplantation. Heart Vessels 2016;31:722-33.

51. Daneshmand MA, Krishnamoorthy A, Samsky MD, et al. Comparison of 2-Year Outcomes of Extended Criteria Cardiac Transplantation Versus Destination Left Ventricular Assist Device Therapy Using Continuous Flow. Am J Cardiol 2015;116:573-9.

52. John R, Holley CT, Eckman P, et al. A Decade of Experience With Continuous-Flow Left Ventricular Assist Devices. Semin Thorac Cardiovasc Surg 2016;28:363-75.

53. Raichlin E, Baibhav B, Lowes BD, et al. Outcomes in Patients with Severe Preexisting Renal Dysfunction
After Continuous-Flow Left Ventricular Assist Device Implantation. ASAIO J 2016;62:261-7.

54. Sileshi B, O'Hara BK, Davis ME, et al. Outcomes of Patients Implanted Using a Left Thoracotomy Technique for a Miniaturized Centrifugal Continuous-Flow Pump. ASAIO J 2016;62:539-44.

55. Xuereb L, Go PH, Kaur B, et al. Impact of Preoperative Atrial Fibrillation on Postoperative Thromboembolic Events After Left Ventricular Assist Device Implantation. Ann Thorac Surg 2016;102:1543-9.

56. Centofanti P, Baronetto A, Attisani M, et al. Thrombosis in left ventricular assistance device with centrifugal technology: is early thrombolysis a better solution? Int J Artif Organs 2017;40:629-35.

57. Hanke JS, Rojas SV, Cvitkovic T, et al. First results of HeartWare left ventricular assist device implantation with tunnelling of the outflow graft through the transverse sinus. Interact Cardiovasc Thorac Surg 2017;25:503-8.

58. Steffen RJ, Blackstone EH, Smedira NG, et al. Optimal Timing of Heart Transplant After HeartMate II Left Ventricular Assist Device Implantation. Ann Thorac Surg 2017;104:1569-76.

59. Tozzi P, Banfi C, Ahmadov K, et al. HeartMate 3 in Lowest INTERMACS Profile Cohort: The Swiss Experience. ASAIO J 2017;63:752-8.

60. Carrozzini M, Bejko J, Gambino A, et al. Results of newgeneration intrapericardial continuous flow left ventricular assist devices as a bridge-to-transplant. J Cardiovasc Med (Hagerstown) 2018;19:739-47.

61. Tahsili-Fahadan P, Curfman DR, Davis AA, et al. Cerebrovascular Events After Continuous-Flow Left Ventricular Assist Devices. Neurocrit Care 2018;29:225-32.

62. Volkovicher N, Kurihara C, Critsinelis A, et al. Outcomes in patients with advanced heart failure and small body size undergoing continuous-flow left ventricular assist device implantation. J Artif Organs 2018;21:31-8.

63. Yin MY, Ruckel S, Kfoury AG, et al. Novel Model to Predict Gastrointestinal Bleeding During Left Ventricular Assist Device Support. Circ Heart Fail 2018;11:e005267.

64. Braun OÖ, Nilsson J, Gustafsson F, et al. Continuousflow LVADs in the Nordic countries: complications and mortality and its predictors. Scand Cardiovasc J 2019;53:14-20.

65. Jorde UP, Shah AM, Sims DB, et al. ContinuousFlow Left Ventricular Assist Device Survival Improves With Multidisciplinary Approach. Ann Thorac Surg 2019;108:508-16.

66. Kyvernitakis A, Pappas O, Farmakiotis D, et al. 
Bloodstream Infections in Continuous Flow Left Ventricular Assist Device Recipients: Diagnostic and Clinical Implications. ASAIO J 2019;65:798-805.

67. John R, Naka Y, Smedira NG, et al. Continuous flow left ventricular assist device outcomes in commercial use compared with the prior clinical trial. Ann Thorac Surg 2011;92:1406-13; discussion 1413.

68. Goldstein DJ, Meyns B, Xie R, et al. Third Annual Report from the ISHLT Mechanically Assisted Circulatory Support Registry: A comparison of centrifugal and axial continuous-flow left ventricular assist devices. J Heart Lung Transplant 2019;38:352-63.

69. Kormos RL, Cowger J, Pagani FD, et al. The Society of Thoracic Surgeons Intermacs database annual report: Evolving indications, outcomes, and scientific partnerships. J Heart Lung Transplant 2019;38:114-26.

70. Wever-Pinzon O, Selzman CH, Drakos SG, et al. Pulsatility and the risk of nonsurgical bleeding in patients supported with the continuous-flow left ventricular assist device HeartMate II. Circ Heart Fail 2013;6:517-26.

71. Uriel N, Pak SW, Jorde UP, et al. Acquired von Willebrand syndrome after continuous-flow mechanical device support contributes to a high prevalence of bleeding during long-term support and at the time of transplantation. J Am Coll Cardiol 2010;56:1207-13.

72. Kirklin JK, Naftel DC, Kormos RL, et al. Fifth INTERMACS annual report: risk factor analysis from more than 6,000 mechanical circulatory support patients. J Heart Lung Transplant 2013;32:141-56.

73. Mudd JO, Cuda JD, Halushka M, et al. Fusion of aortic valve commissures in patients supported by a continuous axial flow left ventricular assist device. J Heart Lung Transplant 2008;27:1269-74.

74. Baumwol J, Macdonald PS, Keogh AM, et al. Right heart failure and "failure to thrive" after left ventricular assist device: clinical predictors and outcomes. J Heart Lung Transplant 2011;30:888-95.

75. Green CP, Porter CB, Bresnahan DR, et al. Development and evaluation of the Kansas City Cardiomyopathy Questionnaire: a new health status measure for heart failure. J Am Coll Cardiol 2000;35:1245-55.

76. Rector TS, Cohn JN. Assessment of patient outcome with the Minnesota Living with Heart Failure questionnaire: reliability and validity during a randomized, doubleblind, placebo-controlled trial of pimobendan. Am Heart J 1992;124:1017-25.

77. Stulak JM, Davis ME, Haglund N, et al. Adverse events in contemporary continuous-flow left ventricular assist devices: A multi-institutional comparison shows significant differences. J Thorac Cardiovasc Surg 2016;151:177-89.

78. McIlvennan CK, Magid KH, Ambardekar AV, et al. Clinical outcomes after continuous-flow left ventricular assist device: a systematic review. Circ Heart Fail 2014;7:1003-13.

79. Yoshioka D, Sawa Y. Clinical results of continuous-flow left ventricular assist device(LVAD) for severe heart failure patients. Kyobu Geka 2013;66:57-61.

80. Miller LW, Pagani FD, Russell SD, et al. Use of a continuous-flow device in patients awaiting heart transplantation. N Engl J Med 2007;357:885-96.

81. Zierer A, Melby SJ, Voeller RK, et al. Late-onset driveline infections: the Achilles' heel of prolonged left ventricular assist device support. Ann Thorac Surg 2007;84:515-20.

82. Schulman AR, Martens TP, Christos PJ, et al. Comparisons of infection complications between continuous flow and pulsatile flow left ventricular assist devices. J Thorac Cardiovasc Surg 2007;133:841-2.

83. John R, Kamdar F, Eckman P, et al. Lessons learned from experience with over 100 consecutive HeartMate II left ventricular assist devices. Ann Thorac Surg 2011;92:15939; discussion 1599-600.

84. Stern DR, Kazam J, Edwards P, et al. Increased incidence of gastrointestinal bleeding following implantation of the HeartMate II LVAD. J Card Surg 2010;25:352-6.

85. Slaughter MS, Pagani FD, Rogers JG, et al. Clinical management of continuous-flow left ventricular assist devices in advanced heart failure. J Heart Lung Transplant 2010;29:S1-39.

86. Morgan JA, Paone G, Nemeh HW, et al. Gastrointestinal bleeding with the HeartMate II left ventricular assist device. J Heart Lung Transplant 2012;31:715-8.

87. Crow S, John R, Boyle A, et al. Gastrointestinal bleeding rates in recipients of nonpulsatile and pulsatile left ventricular assist devices. J Thorac Cardiovasc Surg 2009;137:208-15.

88. Cannegieter SC, Rosendaal FR, Briët E. Thromboembolic and bleeding complications in patients with mechanical heart valve prostheses. Circulation 1994;89:635-41.

89. Hayes HM, Dembo LG, Larbalestier R, et al. Management options to treat gastrointestinal bleeding in patients supported on rotary left ventricular assist devices: a single-center experience. Artif Organs 2010;34:703-6.

90. Crow S, Chen D, Milano C, et al. Acquired von Willebrand syndrome in continuous-flow ventricular assist device recipients. Ann Thorac Surg 2010;90:1263-9; discussion 1269. 
91. Geisen U, Heilmann C, Beyersdorf F, et al. Non-surgical bleeding in patients with ventricular assist devices could be explained by acquired von Willebrand disease. Eur J Cardiothorac Surg 2008;33:679-84.

92. Crow S, Milano C, Joyce L, et al. Comparative analysis of von Willebrand factor profiles in pulsatile and continuous left ventricular assist device recipients. ASAIO J 2010;56:441-5.

93. Tsai HM, Sussman II, Nagel RL. Shear stress enhances the proteolysis of von Willebrand factor in normal plasma. Blood 1994;83:2171-9.

94. Siedlecki CA, Lestini BJ, Kottke-Marchant KK, et al. Shear-dependent changes in the three-dimensional structure of human von Willebrand factor. Blood 1996;88:2939-50.

95. Klovaite J, Gustafsson F, Mortensen SA, et al. Severely impaired von Willebrand factor-dependent platelet aggregation in patients with a continuous-flow left ventricular assist device (HeartMate II). J Am Coll Cardiol 2009;53:2162-7.

Cite this article as: $\mathrm{McNamara} \mathrm{N}$, Narroway H, Williams M, Brookes J, Farag J, Cistulli D, Bannon P, Marasco S, Potapov E, Loforte A. Contemporary outcomes of continuousflow left ventricular assist devices-a systematic review. Ann Cardiothorac Surg 2021;10(2):186-208. doi:10.21037/acs-2021cfmcs-35
96. John R, Lee S. The biological basis of thrombosis and bleeding in patients with ventricular assist devices. J Cardiovasc Transl Res 2009;2:63-70.

97. Letsou GV, Shah N, Gregoric ID, et al. Gastrointestinal bleeding from arteriovenous malformations in patients supported by the Jarvik 2000 axial-flow left ventricular assist device. J Heart Lung Transplant 2005;24:105-9.

98. Demirozu ZT, Radovancevic R, Hochman LF, et al. Arteriovenous malformation and gastrointestinal bleeding in patients with the HeartMate II left ventricular assist device. J Heart Lung Transplant 2011;30:849-53.

99. Teuteberg JJ, Cleveland JC Jr, Cowger J, et al. The Society of Thoracic Surgeons Intermacs 2019 Annual Report: The Changing Landscape of Devices and Indications. Ann Thorac Surg 2020;109:649-60.

100. Topkara VK, Kondareddy S, Malik F, et al. Infectious complications in patients with left ventricular assist device: etiology and outcomes in the continuous-flow era. Ann Thorac Surg 2010;90:1270-7. 